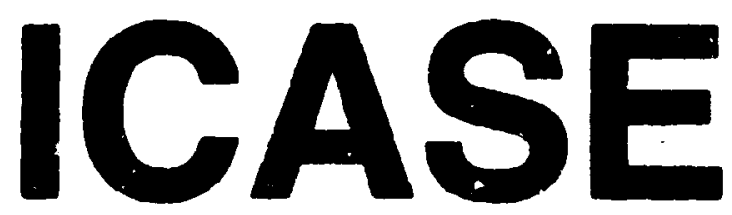

\title{
A PDE SENSITIVITY EQUATION METHOD FOR OPTIMAL AERODYNAMIC DESIGN
}

\section{Jeff Borggaard John Burns}

NASA Contract No. NASI 19480 June 1996

Institute for Computer Applications in Science and Engineering NASA Langley Research Center

Hampion, VA 23681-0001

Operated by Universities Space Research Association

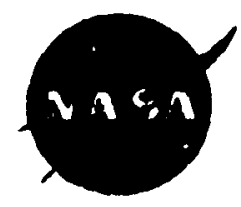

National Aeronautics and Space Administration

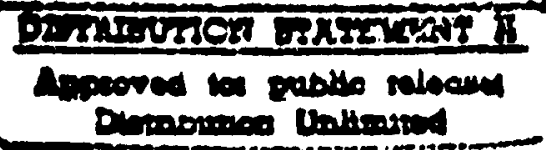

\section{Langley Research Center}

Hampton, Virginia 23681-0001 


\title{
A PDE Sensitivity Equation Method for Optimal Aerodynamic Design
}

\author{
Jeff Borgsaard and John Burns" \\ Interdisciplinary Center for Applied Mathematics \\ Virginia Tech \\ Blacksburg, VA 24061-0531
}

\begin{abstract}
Abatract

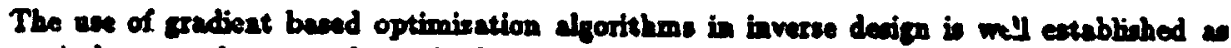

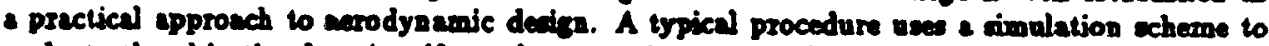
evaluate the objective fenction (broen the approximate atates) and its cradient, then pares this information to in optimisation lyorishm. Onca the mimulation echene (CPD fow colver) ha been selected and need to provide approuimate fuction ovalextions, there are cevard posable epprosecher to the problem of competing gredients. One popular method is to difierentive

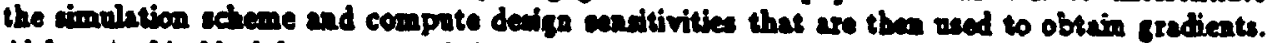
Althow gh this black-box appsosch he many advantege in shepe optimisation problems, one

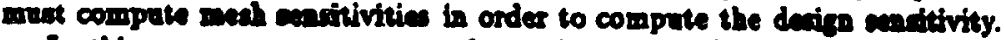

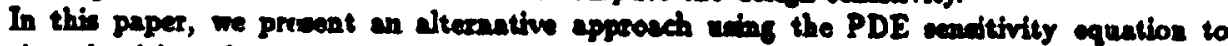

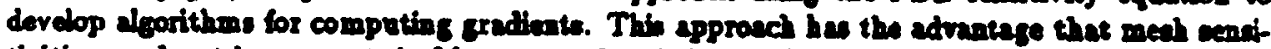
tivities need not be comprted. Moreover, when it is ponible to we the CPD scheme for both the forward problese asd the menitivity equation, then there are comprtatboud advantages. Al apparent dindvantage of this approsch is that if does sot alwas produce consintent derive. tives. Bowmer, for a proper combination of decretisation schemes, one can show esymptotic

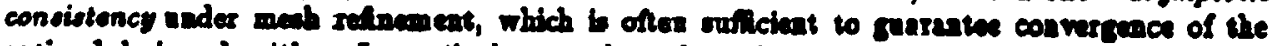

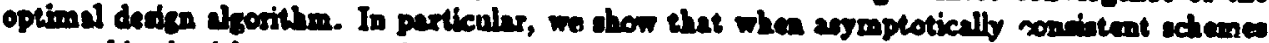

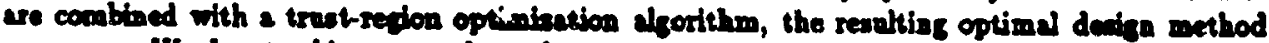
converges. We denote this opprosech so the eensitivity epration method.

The curitivity equation method is preantod, convergence reaulte are given and the approsch is illustrated on two optined deaign probions trolving ahocks.
\end{abstract}

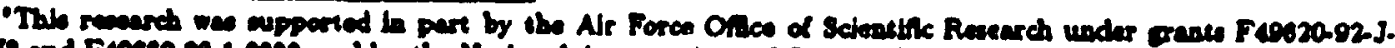

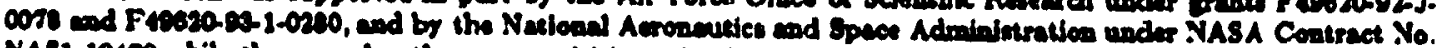

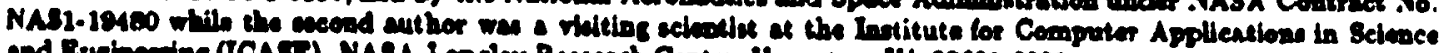

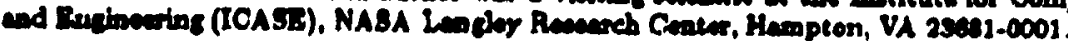




\section{Introduction}

Optimal design problems consist of selecting design parameters for a system in order to optimize a given design objective, usually constrained to satiofy a partial differential equation. In many of these problems, design parameters describe the shape of an object. Examples of theac shape optimization problems include drag reduction [21], [22], weight minimization [14], optimal sensor/actustor placement [6], airfoil design [16], [17], [18], [19] and the design of wind tunnel elements [15].

Traditionally, approximate solutions of these problems are found by "cut and try" methods, combining a designer's engineering experience with repeated experimental testing. This is often expensive, motivating computational methods which compute the optimal design directly. These methods require defining an objective function and an appropriate PDE model of the states of the system. A comparison of several optimal design methods may be found in [13].

Many popular approaches couple a gradient-based optimization algorithm with function evaluations provided by a proven simulation scheme. One of the disadvantages of these approaches is the expense of computing the gradient. Tsing finite differences is often too costly, even if appropriate step sizes can be found and the simulation scbeme can take advantage of "aearby" solutions (as is the case with iterative solvers for nonlinear equations).

Two strategies for alleviating the computational expense of gradient evaluations are adjoint variables [17] and design sensitivities [14]. Adjoint methods are advantar geous when either the problem is self-adjoint or there are a large number of design parameters. However, when there are relatively few design parameters, uving decign censitivities, quantities which describe the influence of the design parameters on the atates of the system, is an attractive alternative. In addition to efficient gradient computations, they can be used in some problems to construct an effective update of the approximate Heasian for quasi-Newton optimization algorithms, e.g. [10].

A standard approach often used to compute the design rensitivities is based on (implicitly) differentiating the simulation scheme (for the states) with respect to the design variables. Uning the chain rule to carry out this calculation, results in an efficient numerical scheme for the sensitivities. The efficiency arises from reusing many of the quantities computed in the simulation schetse. In fact, the "inversion" of the system matrix (i.e. the matrix factorization) can often be reused. 
A disadvantage of this approsch is that for shape optimization problems, the discretizstion is parameter dependent. Thus, derivative of the discretization (mesh sensitivities) are required for each shape parameter. Depending on the simulation scheme used for the states, determining the discretization can require the solution of a partial differential equation ( $a$ is the case for finite difference solutions of viscous flow problems (26]). This requires a strategy for computing the mesh sensitivities [20], or for computing an approximation to them [24], [25].

Another approach to finding decigo censitivities relies on approximating the partial differential equation, known as the sensitivity equation. This equation is obtained by implicitly differentiating the (infinite dimeasioial) state equation with respect to each design parameter. As shown in [2], using the same numerical scheme to approximate the sensitivity equation which is used to approximate the states, leads to an efficient scheme with similar computational advantages as the design sensitivity approach deacribed above. Furthermore, since the discretization is applied directly to the sensitivity equations, no sensitivity of the mesh is required. The rensitivity equation is always linear in the deaign sensitivity, even if the state equation is nonlinear. Since there is no requirement to use the same numerical scheme, it is possible to gain additional computational axvinge by using a scheme which takes advantage of the linearity in the sensitivity equations.

An apparent disadvantage of this approach is that it does not compute conoistent derivatives. In other words, the senoitivity equation approach does not capture the sensitivity of the truncation errors in the scheme. Thus, there is a concern that providing an optimization algorithm with an approximation of the gradient of the infinite dimensional objective function instead of the gradient of the approximate objective function would cause the algorithm to fail. One might expect, however, that if the gradients are "close enough" to the true gradients, then the optimization algorithm ahould atill converge. We show that this convergence can be eatabbibed if one combines compatible simulation and optimization schemes.

Trust-region optimization algorithms are constructed to be globally convergent by minimizing a model of the objective function in a region where the model is "trusted". This leads to robust algorithms capable of bandling inaccuracies in the model. In fact, convergence results have been given for these algorithms when the model is based on inaccurate gradient information [7], [8]. The results hold provided the gracients atiofy a given exror condition. Therefore, it is natural to consider an optimal deaign mothod 
which couples a trust-region optimization algorithm with gradients computed using the sensitivity equation approach. We denote thio combined sensitivity/trust-region algorithm by the sensitivity equation method (SEM).

In this work, we present and analyze the sensitivity equation method. The method can be applied to a wide class of optimal design problems, including those mentioned above, however, we focus on the particular example of shape optimization of Euler flows in order to illustrate the method. In the next section, we describe two design problems. In Section 3, we present the sensitivity equation method including the truat-region algorithm and the use of the sensitivity equation to find the design rensitivities. Furthermore, we compare various numerical approximations of the sensitivity equation with approaches based on the discretized equations. Section 4 discusses a number of convergence issues and includes a convergence theorem for the sensitivity equation method. In Section 5, we use a one dimensional duct design problem to describe the implementation of the rensitivity equation approach. Finally, we describe the implementation and perform shape optimization for a two dimensional forebody simulator design problem where the steady-state Euler equations are used to model the state variables.

\section{Illustrative Examples}

We present two optimal design problems below which are used to illustrate the sensitivity equation method. These problems conoist of determining shape parameters which produce a solution to the Euler equations that matches a desired flow "as clocely as possible." The firat problem is motivated by the design of a wind turnel element in order to produce a desired flow in the test section. We study a two dimensional analogue of this problem. The second probjem consiste of prescribing the cross-nectional area of a one dimensional duet to produce $₫$ duct fow which matches a desired flow profile. This problem was used by Frank and Shubin [13] in their atudy of optimal design.

\subsection{Porebody Simulator Deaign Problem}

The Arnold Engineering Development Center (AEDC) operates a free-jet test facility which is uned for full-scale testing of commercial and military aircraft engines. Engines are evaluated for performance and safety under various free dight conditions. While 


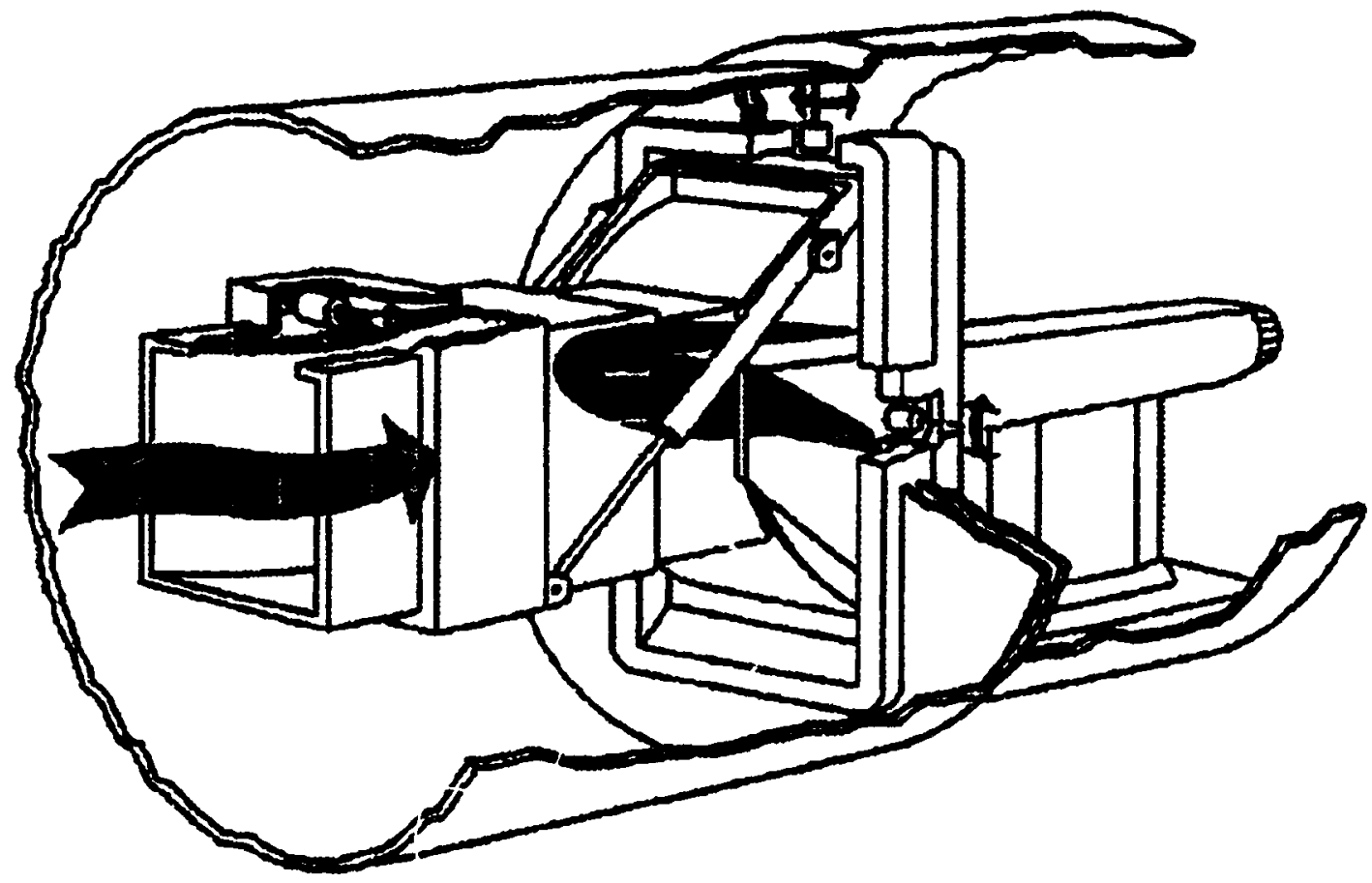

Figure 1: Forebody Simulator Deaiga Problem

this facility is large enough to house engines, it is not large enough to bouse an entire aircraft forebody. Thus, the effect of the aircraft forebody on the engine inlet flow profile must be simulated. One way of doing this is to replece the actual forebody by a oraller object, called a forebody simulator (FBS). The use of the FBS is illustrated in Figure 1. The FBS design problem is to specify the shape of this FBS so that it produces an engine inlet flow profile which is as close to eame desired profile as possible [15]. The decired profile can be determined by performing either a wind tunnel simulation or a computational simulation of a model configuration resembling a teat condition of the aircraft engine.

In order to demonatrate the applicability of the SEM, we consider a two dimensional analogue of this problem. This problem, depicted in Figure 2, is to find the shape of the curve $\Gamma$, which produce an outflow that matchee the outflow generated by the original (longer) forebody a clonely a possible. The flow, $Q$ (consisting of the density $\rho$, the momentum $p u \hat{i}+p v \hat{j}$ and the sum of the internal and kinetic energy $E)$ is modeled uring the steady state Euler equations, 


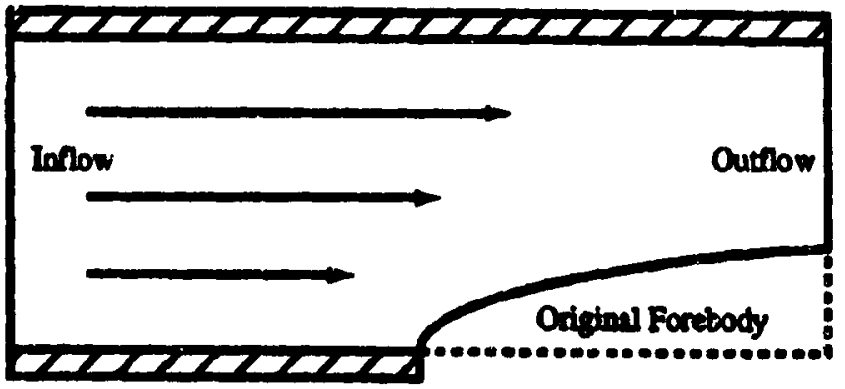

Long Forobody (Din to be Marched)

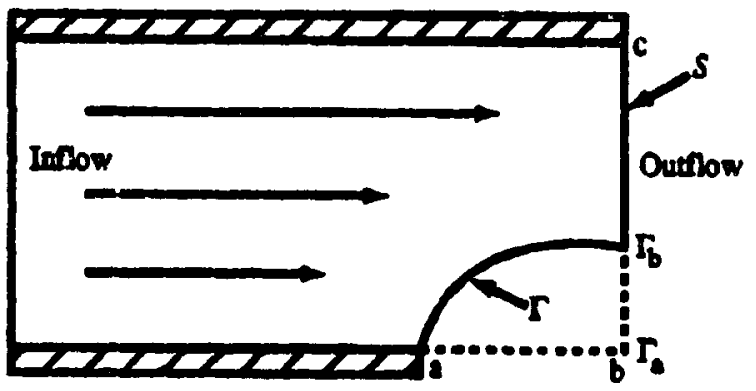

Sharesed Forebody (Shape to be Determined)

Figure 2: 2D Forebody Simulator Deaign Problem

$$
\frac{\partial F}{\partial x}+\frac{\partial G}{\partial y}=0
$$

where

$$
F=u Q+\left[\begin{array}{c}
0 \\
P \\
0 \\
P u
\end{array}\right], \quad G=v Q+\left[\begin{array}{c}
0 \\
0 \\
P \\
P v
\end{array}\right] \text { and } Q=\left[\begin{array}{c}
\rho \\
\rho u \\
\rho v \\
E
\end{array}\right] \text {. }
$$

The preasure $P$ is related to the elements of $Q$ by

$$
P=(\gamma-1)\left[\Sigma-\frac{1}{2} \rho\left(u^{2}+v^{2}\right)\right],
$$

where $\gamma$ is the ratio of opecific heats $(\gamma=1.4$ for air). Given a forebody oimulator shape $\Gamma$, the flow $Q(\Gamma)$ is determined by solvigg the Euler equations (1) in the test cell domain $\Omega(\Gamma)$ subject to the boundary conditions (for oupersonic flow):

$$
\begin{aligned}
Q & =Q_{b} \text { at the test cell inflow, } \\
(u, v) \cdot \hat{n} & =0 \text { and } \\
\frac{\partial}{\partial n}((u, v) \cdot \hat{t}) & =0 \text { at the walls (no flow penetration), }
\end{aligned}
$$


where $\hat{n}$ and $\hat{t}$ are the normal and tangential vectors at the boundary, respectively. The set of admissible forebody vimulator shapes is

$$
\mathcal{A}=\left\{\Gamma \in C^{1}(a, b) \mid \Gamma(a)=\Gamma_{a}, \Gamma(b)=\Gamma_{b} \text { and } \Gamma(x) \geq \Gamma_{a}, \forall x \in(a, b)\right\} .
$$

A statement of the design problem is given below.

Problem 2.1 (Forebody Simulator Design) Let $Q$ be a desired flow at the outflow (engine inlet),

$$
S=\left\{(x, y) \mid x=b, \Gamma_{b} \leq y \leq c\right\} .
$$

Define the objective function

$$
\mathcal{J}(\Gamma)=\int_{S}\|Q(\Gamma)-Q\|_{2}^{2} d S,
$$

where $Q(\Gamma)$ represents the solution of (1) with boundary conditions (4).(6) in the test cell $\Omega(\Gamma)$. The forebody simulator design problem is to find $\Gamma . \in \mathcal{A}$ such that

$$
\mathcal{J}\left(\Gamma_{*}\right) \leq \mathcal{J}(\Gamma) \text { for all } \Gamma \in \mathcal{A} \text {. }
$$

Closed form solutions to (1) with (4)-(6) are araiable only for special domains. Therefore, we consider approximate solutions of (1) and bence the approximation of Problem 2.1.

The discretization is performed by selecting mesh points in the flow domain $\Omega(\Gamma)$ where the flow variables will be approximated. It is desirable to select this mesh in such a way that the points are more dense in regions where flow gradients are expected to be "large" (in order to have more accurate differencing) and more coarse in regions where the flow is nearly constant (in order to save computer time). Other issues. ouch as celecting points with no aharp changes in density and with sufficient resolution to treat the boundary conditions, make the mesh generation a science in and of itself (see e.g. [26]).

Another conatraint on the diacretization, to nimplify the implementation of a firite difference acheme, is to use a regular meah, i.e. a mesh where there exists a bijective map taking the meab points to a lattice of points in the computational space. For example, suppose that $\mathcal{M}$ is $\& C^{1}$ misping,

$$
\mathcal{M}:(x, y) \rightarrow(\xi, \eta),
$$

then derivatives in the physical opace are axily approximated on the lattice using the chain rule. Denoting the Jacobian of the mapping by $J_{M}$, the tranaformed Euler 
equations become,

$$
\frac{\partial \bar{F}}{\partial \xi}+\frac{\partial \bar{G}}{\partial \eta}=0
$$

where

$$
\begin{aligned}
& F=U \bar{Q}+P J_{M}^{-1}\left[\begin{array}{c}
0 \\
\frac{\partial \xi}{\partial x} \\
\frac{\partial \xi}{\partial v} \\
U
\end{array}\right], \quad G=V \bar{Q}+P J_{M}^{-1}\left[\begin{array}{c}
0 \\
\frac{\partial \eta}{\partial z} \\
\frac{\partial \eta}{\partial y} \\
V
\end{array}\right], \\
& \bar{Q}=J_{M}^{-1} Q, \quad U=\frac{\partial \xi}{\partial x} u+\frac{\partial \xi}{\partial v} v \text { and } \quad V=\frac{\partial \eta}{\partial x} u+\frac{\partial \eta}{\partial y} v .
\end{aligned}
$$

A standard finite difference scheme, developed by Beam and Warming [1] is used to approximate the trassformed equations. The scheme introduces a time variable, $t$ as a means of iterating an initial guess for the solution, to a solution of the steady state equations. Second ano fourth order artificial dissipation terms are added for stability, represented by $\Psi^{(2)}$ and $\Psi^{(4)}$, respectively. This scheme is implemented in the PARC2D code [9]. Several implementation issues are discussed briefly below which are referred to in later sections. Readers intereated in more code details or the actual expressions used for $\Psi^{(2)}$ and $\Psi^{(1)}$, should consult [9].

The difference scheme produces a system of equations for the update of the flow variables, $\Delta \bar{Q}^{n}$. Thus, the solution at the nth iteration, $\bar{Q}^{n}$ is determined from

$$
Q^{n}=Q^{n-1}+\Delta Q^{n-1}
$$

The syatem matrix produced by the approximation above is quite large due to differencing in each direction. However, this problem is circumvented using an approximate factorization into a product of two matrices, each corresponding to differencing in one of the lattice directions. The final system bas the form:

$$
\begin{aligned}
{\left[I+\Delta t \delta_{l} \bar{A}^{n}-\nabla_{l}\left(\Psi_{l}^{(2)}+\Psi_{l}^{(1)}\right)\right.} & \left.\Delta_{l} J_{M}\right] \times \\
{\left[I+\Delta t \delta_{\eta} \bar{B}^{n}-\nabla_{\eta}\left(\Psi_{\eta}^{(2)}+\Psi_{\eta}^{(4)}\right)\right.} & \left.\Delta_{\eta} J_{M}\right] \Delta Q^{n}= \\
& -\Delta t \delta_{l} F^{n}-\Delta t \delta_{\eta} \bar{G}^{n} \\
& +\Delta t \nabla_{l}\left(\Psi_{l}^{(2)}-\Psi_{\zeta}^{(1)} \Delta_{l} \nabla_{l}\right) \Delta_{\zeta}\left(J_{M} Q^{n}\right) \\
& +\Delta t \nabla_{\eta}\left(\Psi_{\eta}^{(2)}-\Psi_{\eta}^{(4)} \Delta_{\eta} \nabla_{\eta}\right) \Delta_{\eta}\left(J_{M} Q^{n}\right)
\end{aligned}
$$

where

$$
A^{n}=\frac{\partial F}{\partial Q^{n}}\left(Q^{n}\right) \quad \text { and } \quad B^{n}=\frac{\partial G^{n}}{\partial Q}\left(Q^{n}\right)
$$


The subrcripted terms $\delta, \nabla$ and $\Delta$ represent the central, backward and forward difference operators, respectively, in the lattice direction indicated by the subscript. The converged solution is denoted by $Q^{N}(x, y) \equiv Q^{n_{*}}(M(x, y))$.

We introduce Bezier curves to parameterize the forebody simulator. Bezier polynomials possess several nice properties when used in approximations. The most important for the examples presented bere are the convex hull and endpoint interpolation properties (see e.g. Farin [12]). For this problem, we consider a set of two parameter, $q=\left(q^{1}, q^{2}\right)$, Bezier curves

$$
B=\left\{\Gamma \in C^{1}[0,1] \mid \Gamma(s)=\left(\Gamma_{x}(s), \Gamma_{y}(s ; q)\right), \Gamma_{y}(s ; q) \geq \Gamma_{s}, s \in[0,1], q \in \mathbb{R}^{2}\right\}
$$

where

$$
\begin{aligned}
\Gamma_{x}(s) & =a B_{0,3}(s)+0.6 B_{1,3}(s)+0.8 B_{2,3}(s)+b B_{3,3}(s) \\
\Gamma_{y}(s ; q) & =\Gamma_{a} B_{0,3}(s)+q^{1} B_{1,3}(s)+q^{2} B_{2,3}(s)+\Gamma_{b} B_{3,3}(s)
\end{aligned}
$$

and

$$
B_{i, r}(x)=\left(\begin{array}{c}
r \\
i
\end{array}\right) x^{i}(1-x)^{r-i} .
$$

We also aysume $a=0.5$ and $b=1.0$. We can now introduce the approximate forebody simulator design problem.

Problem 2.2 (Approximate Forebody Simulator Design) Let $\left\{\hat{Q}_{i}\right\}_{i=1}^{g}$ be desired flow measurements at $S$. We asume that the date measurements are given at the quadrature points, otherwise interpolation must be used. Define the objective function

$$
J_{i}^{N}(\Gamma)=\sum_{i=1}^{6} c_{i}\left\|Q^{N}\left(x_{i} ; \Gamma\right)-Q_{i}\right\|_{2}^{2},
$$

where $Q^{N}\left(x_{i} ; \Gamma\right)$ represents the approximate solution to $(1)$ in the domain $\Omega(\Gamma)$ at the quadrature point $x_{i}$. The approximate forebody simulator design problem is to find $\Gamma, \in B$ such that

$$
\mathcal{J}_{0}^{N}\left(\Gamma_{-}\right) \leq \mathcal{J}_{0}^{N}(\Gamma) \text { for all } \Gamma \in B
$$

Let

$$
\mathcal{Q}=\left\{\left(q^{2}, q^{2}\right) \in \mathbb{R}^{2} \mid \Gamma\left(\cdot ; q^{2}, q^{2}\right) \in B\right\},
$$

then the problem can be equivalently stated as finding $\left(q_{*}^{1}, q_{n}^{2}\right) \in \mathcal{Q}$ such that

$$
J_{g}^{N}\left(q_{*}^{1}, q_{*}^{2}\right) \leq \mathcal{J}_{j}^{N}\left(q^{1}, q^{2}\right) \text { for ail }\left(q^{1}, q^{2}\right) \in Q \text {. }
$$




\subsection{Duct Design Problem}

This problem consists of designing the cross-sectional area of 2 one-dimensional duct such that, under specified inlet and outlet conditions, it produces a flow which is as close to a desired transonic flow as poesible. The governing conservation laws (steady state continuity, momentum and energy equations) can be reduced to a single twopoint boundary value problem (BVP) for the velocity. It was shown in [13] that the velocity $u$, is the solution of

$$
\begin{gathered}
\frac{\partial}{\partial x} f(u)+g(u, A)=0, \\
u(0)=u_{\text {in }} \text { and } u(1)=u_{\text {ouc }},
\end{gathered}
$$

where $u_{\text {in }}$ and $u_{\text {out }}$ are the velocities at the inlet and outlet of the duct, $A$ is the cross-sectional area of the duct,

$$
f(u)=u+\frac{\bar{H}}{u}, \quad g(u, A)=\frac{1}{A}\left(\frac{\partial}{\partial x} A\right)\left(\bar{\gamma} u-\frac{\bar{H}}{u}\right) \quad \text { and } \bar{\gamma}=\frac{\gamma-1}{\gamma+1},
$$

where $\tilde{H}$ and $\gamma$ are flow constants taken to be 1.14 and 1.4, respectively. The RankineHugoniot condition yields the speed of cound as $u_{0}=\sqrt{H}$. Unique solutions of this BVP are guaranteed for monotone area functions, therefore, crose-sectional areas, $A$, are restricted to

$$
\mathcal{A}=\left\{A \in C^{1}(0,1) \mid A(0)=A_{\mathrm{h}}, A(1)=A_{\text {out }} \text { and } \frac{\partial}{\partial x} A(x)>0, \forall x \in(0,1)\right\}
$$

for fixed inlet and outlet areas of $A_{\text {in }}$ and $A_{\text {out }}$. We now describe the optimal design problem.

Problem 2.3 (Duct Design) Let $\hat{u}(\cdot) \in L^{2}(0,1)$ be a desired transonic flow profile for the duct and define the objective function by

$$
\mathcal{J}(A)=\int_{0}^{1}[u(x ; A)-\hat{u}(x)]^{2} d x
$$

where $u(; A)$ is the solution to (26) corresponding to $A$. The optimal design problem is to find an $A . \in \mathcal{A}$ auch that

$$
\mathcal{J}\left(A_{-}\right) \leq \mathcal{J}(A) \text { for all } A \in \mathcal{A} \text {. }
$$

While the BVP has a closed form solution [13], we consider approximations of (26) and consequently of Problern 2.3 in order to study the more general case. We 
begin by discretizing the duct length into $N$ cells (of length $h=\frac{1}{N}$ ) with centers, $x_{j}=\left(j-\frac{1}{2}\right) h, j=1, \ldots, N$ and define $u_{j}^{N}$ to be the average velocity in the $j$ th cell, i.e.

$$
u_{j}^{N}(A)=\frac{1}{h} \int_{x_{j}-\frac{1}{2}}^{x_{j}+\frac{A}{2}} u(x ; A) d x .
$$

A system of nonlinear equations for $u^{N}(A)=\left\{u_{j}^{N}(A)\right\}_{j=1}^{N}$ can be found by integrating (26) over each cell,

$$
\frac{f\left(u\left(x_{j}+\frac{h}{2} ; A\right)\right)-f\left(u\left(x_{j}-\frac{h}{2} ; A\right)\right)}{h}+g\left(u_{j}^{N}(A), A\left(x_{j}\right)\right)=0, \quad j=1, \ldots, N,
$$

where it was assumed that $\frac{1}{A} \frac{\theta}{\partial z} A$ was nearly constant over each cell. An approximation to $u^{N}$ is found by replacing the fluxes at the cell edges, $f\left(u\left(x_{j}+\frac{h}{2}\right)\right)$, using the cell center values $f_{j}=f\left(u_{j}^{N}\right)$ and $f_{j+1}=f\left(u_{j+1}^{N}\right)$. Two standard first order "Godunov typen methods are the Enquist-Osher scheme

$$
f\left(u\left(z_{j}+\frac{h}{2}\right)\right) \approx F_{j+1 / 2}^{E O}= \begin{cases}f_{j+1} & u_{j}^{N}, u_{j+1}^{N} \leq u_{o} \\ f_{j} & u_{j}^{N}, u_{j+1}^{N} \geq u_{j} \\ f\left(u_{0}\right) & u_{j}^{N}<u_{0}<u_{j+1}^{N} \\ f_{j}+f_{j+1}-f\left(u_{s}\right) & u_{j+1}^{N}<u_{0}<u_{j}^{N}\end{cases}
$$

and the artificial viscosity scheme

$$
f\left(u\left(x_{j}+\frac{h}{2}\right)\right) \approx F_{j+1 / 2}^{A V}=\frac{1}{2}\left(f_{j+1}+f_{j}-\alpha\left(u_{j+1}-u_{j}\right)\right),
$$

where $\alpha$ has been selected as 1 for this study. These approximations were used in [13], but are included above for completeness.

We turn now to the approximation of the cross-sectional area $A$. The space $A$ is replaced by a subset of Bezier quadratic polynomials. The properties of Bezier polynomials allow us to eavily impose both the monotonicity requirement and the matching of inflow and outflow cross-sectional areas. Consider

$$
\begin{array}{r}
B=\left\{A \in C^{1}(0,1) \mid A(x)=A_{1 \mathrm{R}} B_{0,2}(x)+q B_{1,2}(x)+A_{\text {out }} B_{2,2}(x) ;\right. \\
\left.x \in(0,1), q \in\left[A_{\text {in }}, A_{\text {out }}\right]\right\},
\end{array}
$$

where $B_{i, r}$ is defined in (21). Thus, $B$ is a one parameter set of curves in $\mathcal{A}$. We reatrict our optimization problem to this set $B$.

Our final step in the approximation of Problem 2.3 is replacing the integral by a quadrature rule, with the set of quadrature weights and pointa $\left\{\left(c_{i}, x_{i}\right)\right\}_{i=1}^{q}$. We now state the approximate design problem. 
Problem 2.4 (Approximate Duct Design) Let $\left\{\dot{u}_{i}\right\}_{i=1}^{g}$ represent data for a desired traneonic flow profile in the duet. We assume that the date and the approximate solution are given at the quadrature points, otherwise interpolation must be used. Define the objective function

$$
\mathcal{J}_{g}^{N}(A)=\sum_{i=1}^{\infty} c_{i}\left[u_{i}^{N}(A)-\hat{u}_{i}\right]^{2}
$$

where $u^{N}(A)$ is an appraximate solution to (26) with the cross-sectional area $A$. The approximate design problem is to find an $A_{0} \in B$ such that

$$
\mathcal{J}_{g}^{N}\left(A_{-}\right) \leq \mathcal{J}_{g}^{N}(A) \quad \text { for all } A \in \mathcal{B} \text {. }
$$

Note that we can identify any $A \in B$ with the parameter $q \in \mathcal{Q} \equiv\left[A_{\text {in }}, A_{\text {oue }}\right]$ whicb uniquely represents it. Thus we can equivalently state the problem as to find $q_{*} \in \mathcal{Q}$ such that

$$
\mathcal{J}_{o}^{N}(q \cdot) \leq \mathcal{J}_{o}^{N}(q) \quad \text { for all } q \in \mathcal{Q} \text {. }
$$

\section{Sensitivity Equation Method}

\subsection{Trust-Region Algorithms}

We shall use a trust-region algorithm for the optimization lonp. The reason for selecting this type of scheme will be clear when we discuss the convergence properties in Section 4. This is a well known algoritbm. However, we give a brief description below in order to prepare for the formulation of the sensitivity equation method.

The quasi-Newton optimization algorithm produces a sequence of iterates which are obtained by minimizing a local quadratic model of the objective function. This model is constructed using the evaluation of the objective function $\mathcal{J}_{0}^{N}\left(q_{k}\right)$, its gradient $\nabla J_{g}^{N}\left(g_{k}\right)$ and a secant approximation to its Hessian, $H_{k}$ at the current iterate $q_{k}$. The minimization of this model produces the next iterate $q_{k+1}$, i.e.

$$
m_{k}\left(q_{k+1}\right)=\min _{\delta_{k}} m_{k}\left(q_{k}+s_{k}\right)=\min _{\delta_{k}}\left(\mathcal{J}_{g}^{N}\left(q_{k}\right)+\nabla \mathcal{J}_{g}^{N}\left(q_{k}\right)^{T} s_{k}+\frac{1}{2} s_{k}^{T} H_{k} s_{k}\right) .
$$

Thus the aext step is

$$
q_{k+1}=q_{k}-H_{k}^{-1} \nabla \mathcal{J}_{j}^{N}\left(q_{k}\right)
$$

It is well known that for sufficiently clone initial guesses (and assumptions on the objective function), the iterate converge superlinearly to the minimum, $q_{*}$. 
However, the initial guess may not be in this superlinear region. Thus globalization atrategies are employed to bring the iterates into the superlinear region. It is desirable to choose strategies which reduce to the quasi-Newton algorithm close to the minimum. One such strategy is a trust-region algorithm. In this algorithm, a quantity $\delta$, known as the trust-region radius, is used to measure the region in which the local quadratic model, $m_{k}$, is "trusted" as an approximation of the actual objective function, $\mathcal{J}_{j}^{N}$. Thus, the next iterate, $q_{k+1}$, is now found by minimizing the model in this region, i.e.

$$
m_{k}\left(q_{k+1}\right)=\min _{\left\|o_{k}\right\| \leq \delta_{k}} m_{k}\left(q_{k}+s_{k}\right)
$$

where $\delta_{k}$ is the trust-region radius at the kth iteration.

A beuristic for changing the trust-region radius needs to be developed which increases $\delta_{k}$ when the model prediction is good and decreases $\delta_{k}$ when the model prediction is poor. One such strategy uses the ratio,

$$
\rho_{k}=\frac{\mathcal{J}_{q}^{N}\left(q_{k}\right)-\mathcal{J}_{g}^{N}\left(q_{k+1}\right)}{m_{k}\left(q_{k}\right)-m_{k}\left(q_{k+1}\right)}
$$

which is the ratio of the computed reduction to the reduction predicted by the model. If this ratio is small (or negative), then the model did a poor job of predieting $\mathcal{J}_{g}^{N}$ and the trust-region is decreased. Whereas, if the ratio is near 1 , then the model did very well at predicting $\mathcal{J}_{0}^{N}$ and the trust-region radius is increased.

We present the resulting trust-region algorithm below.

\section{Algorithm 3.1 (Trust-Region)}

Select an initial guess $q_{0} \in \mathcal{Q}$, an initial trust-region radius $\delta_{0}$ and constants $0<\eta_{1}<$ $\eta_{2}<1$ and $0<\gamma_{1}<1<\gamma_{2}$. Compute $\mathcal{J}_{j}^{N}\left(q_{0}\right), \nabla \mathcal{J}_{g}^{N}\left(q_{0}\right)$ and select or initialize $K_{0}$.

Do $k=0,1, \ldots$, until "convergence"

1. Determine the approximate solution $s_{k}$ to equation (40). We chose the optimally constrained book-step method [11] to do this.

2. If $\rho_{k}<\eta_{1}$, then set $\delta_{k+1} \in\left(0, \gamma_{1} \delta_{k}\right)$ and $q_{k+1}=q_{k}, J_{j}^{N}\left(q_{k+1}\right)=J_{a}^{N}\left(q_{k}\right)$, $\nabla \mathcal{J}_{g}^{N}\left(q_{k+1}\right)=\nabla \mathcal{J}_{g}^{N}\left(q_{k}\right)$ and $H_{k+1}=H_{k}$.

3. If $\eta_{1}<p_{k}<\eta_{2}$, then set $j_{k+1} \in\left(0, \delta_{k}\right]$ and $q_{k+1}=q_{k}+s_{k}$. Compute $\mathcal{J}_{j}^{N}\left(q_{k+1}\right)$, $\nabla \mathcal{J}_{g}^{N}\left(q_{h+1}\right)$ and the update $H_{h+1}$. 
4. If $\eta_{2}<\rho_{k}$, then set $\delta_{k+1} \in\left[\delta_{k}, \gamma_{2} \delta_{k}\right]$ and $q_{k+1}=q_{k}+s_{k}$. Compute $\mathcal{J}_{k}^{N}\left(q_{k+1}\right)$, $\nabla \mathcal{J}_{j}^{N}\left(q_{k+1}\right)$ and the update $H_{k+1}$.

Continue

\subsection{Design Seusitivities}

In order to apply a gradient based optimization algorithm, such as the trust-region algorithm described above, we need to consider methods for computing the gradient of $J_{g}^{N}$. In this discussion, we consider finding the gradient of $J_{g}^{N}$ (or a sujtable approximation) with respect $t>$ the single design parameter $q$. This discussion can be easily extended to find the gradient of $\mathcal{J}_{j}^{N}$ with respect to multiple design parameters. A straight forward approach is to use a finite difference approximation, e.g.

$$
\frac{\partial}{\partial q} \mathcal{J}_{g}^{N}(q) \approx \frac{\mathcal{J}_{q}^{N}(q+\Delta q)-\mathcal{J}_{i}^{N}(q)}{\Delta q}
$$

Unfortunately, this approach may not be practical for problems where the approximation of the PDE is computationally expensive, and is overly complex in shape optimization problems due to the necessity of computing mesh sensitivities. One way of alleviating the computational burden is to use design sensitivities, quantities which describe the influence of the design variables on the flow variables. For example, we can directly compute the gradient by differentiating (36) as

$$
\frac{\partial}{\partial q} J_{i}^{N}(q)=2 \sum_{i=1}^{g} c_{i}\left[u_{i}^{N}(q)-\hat{u}_{i}\right] \frac{\partial}{\partial q} u_{i}^{N}(q)
$$

The quantity $\frac{\theta}{\delta_{q}} u^{N}=\left\{\frac{g_{q}}{g_{q}} u_{i}^{N}\right\}_{i=1}^{N}$ is the design sensitivity for the discretized flow $u^{N}$.

There are several ways to compute this sensitjvity. As above, one might use finite differences, yielding the approximation

$$
\frac{\partial}{\partial q} u^{N}\left(x_{i ; q}\right) \approx \frac{u^{N}\left(x_{i ;} q+\Delta q\right)-u^{N}\left(x_{i} ; q\right)}{\Delta q} .
$$

When the discretization is parameter dependent, it is easier to compute this approximation using,

$$
\begin{aligned}
\frac{\partial}{\partial q} u^{N}\left(x_{i} ; q\right) \approx & \frac{u^{N}\left(x_{i}+\frac{g}{q_{i}} \mathcal{M}\left(x_{i}\right) \Delta q ; q+\Delta q\right)-u^{N}\left(x_{i} ; q\right)}{\Delta q} \\
& -\frac{\partial}{\partial x} u^{N}\left(x_{i} ; q\right) \frac{\partial}{\partial q} \mathcal{M}\left(x_{i}\right)
\end{aligned}
$$


in order to avoid interpolating back to the unperturbed mesh. This approach has the advantage that it may be possible to select a step size $\Delta q$ using error estimates for $u^{N}$. However, it is as computationally expenoive as computing finite differences on $\mathcal{J}_{\boldsymbol{s}}^{N}$.

A more efficient approach can be obtained by differentiating the simulation scheme used to approximate the flow (the discrete sensitivity approach). For example, in the FBS design problem, the simulation scheme (16) could be differentiated with respect to $g$, leading to a numerical scheme for terms like $\frac{g}{g} u^{N}$. Since the chain rule must be used to carry this out, the resulting scheme for the sensitivities contains terms similar to those found in the simulation scheme. Thus, the sensitivities can be computed efficiently along with the flow. A disadvantage of this approach is that when the discretization is parameter dependent, as in ahape optimization problems, then derivatives of the discretization (terms like $\frac{g}{q} \mathcal{M}$ ) neod to be considered, see e.g. [20].

An alternative approach is based on differentiating the original flow oquation with respect to the design paraneter and then approximating the resulting sensitivity equation. The result is $\left(\frac{g}{g q} u\right)^{N, M}$, where the superscript $N$ refers to the approximation of the flow equation and the superscript $M$ refers to the approximation of the sensitivity equation. Since this approach intercbanges the order of differentiation and approximation, no mesh ssasitivities are required. Furthermore, it has been shown [2] that applying the same approximation scheme to the sensitivity equation leads to similar computational advantages as the discrete approach described above. Moreover, additional computational savings could be obtained by applying a scheme which takes advantage of the linearity of the sensitivity equation. A potential disadvantage of this approach, bowever, is that in general $\frac{g}{g} u^{N} \neq\left(\frac{g}{g} u\right)^{N, M}$, even if the same approximation scheme is used for both the flow and sensitivity equations.

However, if we consider the gradient of the infinite dimensional objective function,

$$
\frac{\partial}{\partial q} \mathcal{J}(q)=2 \int_{0}^{1}[u(x ; A)-\hat{u}(x)] \frac{\partial}{\partial q} u(x ; A),
$$

then uriag the rensitivity equation approach provides an approximation of this gradient, i.e.

$$
\frac{\partial}{\partial q} \mathcal{J}(q) \approx\left(\frac{\partial}{\partial q} \mathcal{J}\right)_{,}^{N, N}(q)=2 \sum_{i=1}^{\dot{C}} q_{i}\left(u_{i}^{N}(q)-\hat{u}_{i}\right)\left(\frac{\partial}{\partial q} u\right)_{i}^{N, M}(q) .
$$

Thua, we have reasos to expect that this approach could produce feasible gradients 
for the optimization scheme. These two sensitivity approaches are described in detail in later sections wing concrete examples.

\subsection{Sensitivity Equation Method}

The sensitivity equation method couples a trust-region optimization algorithm with gradient evaluations provided by approximating the sensitivity equation. Thus we consider applying Algorithm 3.1 with the following quadratic mudel,

$$
\psi_{k}\left(q_{k+1}\right)=\min _{\left\|k_{k}\right\| \leq \delta_{k}} \psi_{h}\left(q_{k}+s_{k}\right)=\min _{\left\|s_{k}\right\| \delta_{k}}\left(J_{\partial}^{N}\left(g_{k}\right)+g_{k}^{T} \delta_{k}+\frac{1}{2} s_{k}^{T} H_{k} s_{k}\right),
$$

Note that we replace the quadratic model $m_{k}$ by $\psi_{k}$ to emphasize the fact that $\nabla J_{g}^{N}$ is approximated by $g_{k}$, computed as $\left(\frac{q}{f_{k}} \mathcal{J}\right)_{,}^{N, N}\left(q_{k}\right)$.

The intent is to use the robuatness of the trust-region optimization algorithm to compensate for the non-consistent gradients. The reoult is an optimal design method which is often more efficient and considerably eaviex to implement than current meth. ods. In the sections below, we discuss convergence issues and describe the implementation of this method.

\section{Convergence Issues}

Definition 4.1 A numerical scheme is said to produce consistent derivatives with respect to approximations $N$ (for the states) and $M$ (for the sensitivities) if

$$
\frac{\partial}{\partial q} \mathcal{J}_{g}^{N}(\cdot)=\left(\frac{\partial}{\partial q} \mathcal{J}\right)_{,}^{N, M}(\cdot)
$$

This is exactly the case for the discrete sensitivity approach, since one actually defines (computes) $\left(\frac{0}{\partial g} \mathcal{J}\right)_{0}^{N, M}(\cdot)$ to be $\frac{g}{\delta} \mathcal{J}_{0}^{N}(\cdot)$.

Definition 4.2 A numerical scheme is said to produce asymptotically consintent derivatives with respect to approximations $N$ (for the states) and $M$ (for the sensitivities) if

$$
\left|\frac{\partial}{\partial q} \mathcal{J}_{\theta}^{N}(q)-\left(\frac{\partial}{\partial q} \mathcal{J}\right)_{,}^{N, M}(q)\right| \rightarrow 0, \quad \forall q \in Q_{0}
$$

is satisfied the approximations $N$ and $M$ are refined.

We now convider the convergence of the censitivity equation method. To begin with, we assume that the following bypotheses hold, 
(H1) For a given $g_{0}$ in the design space $\mathcal{Q}$, let $\mathcal{Q}_{0}$ be an open convex subset containing the level set of $\mathcal{J}_{g}^{N}$ at $q_{0}$, i.e.

$$
\mathcal{L}_{0}=\left\{q \in \mathcal{Q} \mid \mathcal{J}_{g}^{N}(q) \leq \mathcal{J}_{,}^{N}\left(q_{0}\right)\right\} \subset Q_{0} \subseteq Q .
$$

(H2) $\mathcal{J}_{g}^{N}$ is bounded below

(H3) $\mathcal{J}_{\boldsymbol{s}}^{N}$ is Frechet differentiable on $\mathcal{Q}_{0}$

(H4) The Frechet derivative of $\mathcal{J}_{j}^{N}$, denoted by $\nabla \mathcal{J}_{j}^{N}$, is Lipschitz continuous on $Q_{0}$ with Lipschitz constant $L$, i.e.

$$
\left\|\nabla J_{o}^{N}\left(q^{1}\right)-\nabla J_{q}^{N}\left(q^{2}\right)\right\| \leq L\left\|q^{1}-q^{2}\right\| \quad \forall q^{2}, q^{2} \in Q_{0} .
$$

(H5) The approximate gradient, $g_{k}$ is asymptotically consistent to $\nabla \mathcal{J}_{j}^{N}\left(g_{k}\right)$.

(H6) There exists a constant $c_{1} \in(0,1]$ such that

$$
c_{1}\left\|g_{k}\right\|\left\|s_{k}\right\| \leq\left\langle-g_{k}, s_{k}\right\rangle \leq\left\|g_{k}\right\|\left\|s_{k}\right\| \quad \forall k=1,2, \ldots
$$

(H7) There exiat constants $c_{2}, c_{3} \in(0, \infty)$ ouch that

$$
-c_{2}\langle d, d\rangle \leq\left\langle H_{k} d, d\right\rangle \leq c_{3}\langle d, d\rangle \quad \forall k=1,2 \ldots
$$

The following discunsion parallels the proof given in (7) which treats the use of truat-region algorithms with inexact gradient and function values. This discussicin makes use of the fact that we soek the minimum of $J_{j}^{N}$ and have asymptotically consistent derivatives.

Lemma 4.1 Under asoumptions (H6) and (HY), Algorithm 9.1 produces iterates which satiofy

$$
\psi_{k}\left(q_{k}\right)-\psi_{h}\left(g_{k+1}\right) \geq \frac{1}{2} c_{1}\left\|g_{k}\right\| \min \left\{\delta_{k}, \frac{c_{1}\left\|g_{k}\right\|}{c_{3}}\right\}
$$


Proof Note that aince $\psi_{k}\left(q_{k}\right)=\mathcal{J}_{j}^{N}\left(q_{k}\right)$,

$$
\psi_{k}\left(q_{k}\right)-\psi_{k}\left(q_{k+1}\right)=-\left\langle g_{k}, s_{k}\right\rangle-\frac{1}{2}\left\langle H_{k} s_{k}, s_{k}\right\rangle \text {. }
$$

Now, let $s_{k}=\left\|s_{k}\right\|_{\left\|p_{k}\right\|} \equiv a_{*} d_{k}$, then $a_{*}$ colves

$$
\min _{0 \leq \leq \leq \delta_{k}} a\left\langle g_{k}, d_{k}\right\rangle+\frac{1}{2} a^{2}\left\langle H_{k} d_{k}, d_{k}\right\rangle
$$

We can break this up into two cases, when $\left\langle H_{k} d_{k}, d_{k}\right\rangle \geq 0$ and when $\left\langle H_{k} d_{k}, d_{k}\right\rangle<0$. Case 1: Assume $\left(H_{k} d_{k}, d_{k}\right) \geq \hat{0}$, then either

$$
a_{-}=-\frac{\left\langle g_{k}, d_{k}\right\rangle}{\left\langle H_{k} d_{k}, d_{k}\right\rangle}
$$

in which case

$$
\begin{aligned}
\psi_{k}\left(q_{k}\right)-\psi_{k}\left(q_{k+1}\right) & =\frac{\left\langle g_{k}, d_{k}\right)}{\left(H_{k} d_{k}, d_{k}\right\rangle}\left(g_{k}, d_{k}\right\rangle-\frac{1}{2} \frac{\left(g_{k}, d_{k}\right)^{2}}{\left(H_{k} d_{k}, d_{k}\right\rangle^{2}}\left\langle H_{k} d_{k}, d_{k}\right\rangle \\
& =\frac{1}{2} \frac{\left\langle g_{k}, d_{k}\right)^{2}}{\left(H_{k} d_{k}, d_{k}\right)} \geq \frac{1}{2} c^{2} \frac{\left\|g_{k}\right\|^{2}}{c_{3}}
\end{aligned}
$$

using hypotheses (H6) and (H7), or

$$
a .=\delta_{k}
$$

in which case

$$
\delta_{k}<-\frac{\left\langle g_{k}, d_{k}\right\rangle}{\left\langle H_{k} d_{k}, d_{k}\right\rangle}
$$

implies

$$
\begin{aligned}
\psi_{k}\left(g_{k}\right)-\psi_{k}\left(g_{k+1}\right) & =-\delta_{k}\left\langle g_{k}, d_{k}\right\rangle-\frac{1}{2} \delta_{k}^{2}\left\langle H_{k} d_{k}, d_{k}\right\rangle \\
& \geq-\delta_{k}\left\langle g_{k}, d_{k}\right\rangle+\frac{1}{2} \delta_{k}\left(g_{k}, d_{k}\right\rangle \geq \frac{1}{2} c_{1} \delta_{k}\left\|g_{k}\right\|
\end{aligned}
$$

by hypothesis (H6).

Case 2: Assume $\left(H_{k} d_{k}, d_{k}\right\rangle<0$, then $a_{*}=\delta_{k}$. Therefore

$$
\begin{aligned}
\psi_{k}\left(g_{k}\right)-\psi_{k}\left(q_{k+1}\right) & =-\delta_{k}\left(g_{k}, d_{k}\right)-\frac{1}{2} \delta_{k}^{2}\left(H_{k} d_{k}, d_{k}\right) \\
& \geq-\delta_{k}\left(g_{k}, d_{k}\right) \geq c_{1} \delta_{k}\left\|g_{k}\right\| \geq \frac{1}{2} c_{1} \delta_{k}\left\|g_{k}\right\| .
\end{aligned}
$$


Lemma 4.2 Assume (Hr) holds, then

$$
\liminf _{k \rightarrow \infty}\left\|g_{k}\right\|>0 \text { and } \lim _{k \rightarrow \infty} \delta_{k}=0
$$

imply

$$
\lim _{h \rightarrow \infty}-\frac{\left(s_{k}, g_{k}\right)}{\left\|s_{k}\right\|\left\|g_{k}\right\|}=1 \text {. }
$$

Proof It was shown [11] that, if $\left\|s_{k}\right\|=\delta_{k}$, then the solution to (48) is given by $s\left(\mu_{k}\right)$, where

$$
s(\mu)=-\left(H_{k}+\mu I\right)^{-1} g_{k}
$$

and $\mu_{k}$ is the unique real number that satisfies $\left\|s\left(\mu_{k}\right)\right\|=\delta_{k}$. Therefore, if $\delta_{h} \rightarrow 0$, then $\mu_{k} \rightarrow \infty$ (since $H_{k}$ is bounded, by (H7)). Thus $s_{k} \rightarrow-\mu_{k}^{-1} g_{k}$.

$\Delta$

Lemma 4.3 Let $J_{i}^{N}$ satisfy (HS), (H4) and (HY), then the iterates satisfy

$$
\left[\psi_{k}\left(q_{k}\right)-\psi_{k}\left(q_{k+1}\right)\right]-\left[\mathcal{J}_{l}^{N}\left(q_{k}\right)-\mathcal{J}_{g}^{N}\left(q_{k+1}\right)\right] \leq \frac{1}{2}\left(c_{2}+L\right)\left\|s_{k}\right\|^{2}-\left\langle g_{k}-\nabla J_{g}^{N}\left(q_{k}\right), s_{k}\right\rangle \text {. }
$$

Proof Using the Cauchy-Schwartz inequality and (H3), we obtain

$$
\begin{aligned}
& \mathcal{J}_{0}^{N}\left(q_{k+1}\right)-\mathcal{J}_{0}^{N}\left(g_{k}\right)=\int_{0}^{1}\left\langle\nabla \mathcal{J}_{0}^{N}\left(g_{k}+\lambda s_{k}\right), s_{k}\right\rangle d \lambda \\
& =\left\langle\nabla \mathcal{J}_{0}^{N}\left(q_{k}\right), s_{k}\right\rangle+\int_{0}^{1}\left\langle\nabla \mathcal{J}_{j}^{N}\left(q_{k}+\lambda_{k}\right)-\nabla \mathcal{J}_{j}^{N}\left(q_{k}\right), s_{k}\right\rangle d \lambda \\
& \leq\left\langle\nabla J_{g}^{N}\left(q_{k}\right), s_{k}\right\rangle+\int_{0}^{1}\left\|\nabla \mathcal{J}_{j}^{N}\left(g_{k}+\lambda s_{k}\right)-\nabla J_{j}^{N}\left(q_{k}\right)\right\|\left\|\alpha_{k}\right\| d \lambda .
\end{aligned}
$$

By the Lipsebitz hypothesis (H4),

$$
\begin{aligned}
\mathcal{J}_{j}^{N}\left(q_{k+1}\right)-\mathcal{J}_{j}^{N}\left(q_{k}\right) & \leq\left\langle\nabla \mathcal{J}_{0}^{N}\left(q_{k}\right), s_{k}\right\rangle+\int_{0}^{1} L\left\|\lambda s_{k}\right\|\left\|s_{k}\right\| d \lambda \\
& =\left\langle\nabla \mathcal{J}_{j}^{N}\left(q_{k}\right), s_{k}\right\rangle+\frac{1}{2} L\left\|s_{k}\right\|^{2} .
\end{aligned}
$$

Thus, using (H7),

$$
\begin{aligned}
& {\left[\psi_{k}\left(q_{k}\right)-\psi_{k}\left(q_{k+1}\right)\right]-\left[J_{k}^{N}\left(q_{k}\right)-J_{0}^{N}\left(g_{k+1}\right)\right]} \\
& \leq-\left\langle g_{k}, s_{k}\right\rangle-\frac{1}{2}\left\langle H_{k} s_{k}, o_{k}\right)+\left\langle\nabla J_{0}^{N}\left(q_{k}\right), o_{k}\right\rangle+\frac{1}{2} L\left\|\alpha_{k}\right\|^{2} \\
& \leq-\left\langle g_{k}-\nabla J_{0}^{N}\left(q_{k}\right), o_{k}\right\rangle+\frac{1}{2}\left(c_{2}+L\right)\left\|s_{k}\right\|^{2}
\end{aligned}
$$

which completes the proof.

$\Delta$

18 
Lemma 4.4 Assume $J_{g}^{N}$ satisfies (H2), (HS) and (H4), and essume (H7) holds, then $\nabla \mathcal{J}_{g}^{N}$ is bounded on $\mathcal{L}_{0}$.

Proof Let $c$ be a constant such that $J_{0}^{N}(q) \geq c, \forall q \in \mathcal{Q}_{0}$ (as guaranteed by (H2)). Assume to the contrary that there exists a point $\bar{q} \in \mathcal{L}_{0}$ wuch that

$$
\left\|\nabla \mathcal{J}_{,}^{N}(\bar{q})\right\|^{2}>8 L\left(\mathcal{J}_{,}^{N}\left(g_{0}\right)-c\right) \text {. }
$$

Define $\bar{s}=-\frac{g}{2 L} \nabla \mathcal{J}_{g}^{N}(\bar{q})$, where we chocse $\alpha$ small enough so that $\bar{q}+\bar{s} \in \mathcal{Q}_{0}$. Then

$$
\begin{aligned}
\mathcal{J}_{g}^{N}(\bar{q})-\mathcal{J}_{j}^{N}(\bar{q}+\bar{J}) & =-\int_{0}^{1}\left\langle\nabla \mathcal{J}_{j}^{N}(\bar{q}), \bar{s}\right\rangle d \lambda-\int_{0}^{1}\left\langle\nabla \mathcal{J}_{g}^{N}(\bar{q}+\lambda \bar{s})-\nabla \mathcal{J}_{j}^{N}(\bar{q}), \bar{\delta}\right\rangle d \lambda \\
& \geq \frac{\alpha}{2 L}\left\|\nabla \mathcal{J}_{j}^{N}(\bar{q})\right\|^{2}-\frac{1}{2} L\|\|^{2} \\
& \geq \frac{\alpha}{4 L}\left\|\nabla \mathcal{J}_{a}^{N}(\bar{q})\right\|^{2}\left(1-\frac{\alpha}{2}\right)>\frac{\alpha}{4 L}\left(1-\frac{\alpha}{2}\right)\left[8 L\left(\mathcal{J}_{a}^{N}\left(q_{0}\right)-c\right)\right]
\end{aligned}
$$

This is positive for $\alpha \in(0,2)$, thus $\mathcal{J}_{j}^{N}(\bar{q})>\mathcal{J}_{\hat{q}}^{N}(\bar{q}+\bar{s})$, which implies $\bar{q}+\bar{s} \in \mathcal{L}_{0}$. In addition

$$
\mathcal{J}_{j}^{N}(\bar{q})-\mathcal{J}_{a}^{N}(\bar{q}+\bar{s})>\mathcal{J}_{j}^{N}\left(g_{0}\right)-c
$$

holds, but this is a contradiction since $\bar{q}$ and $\bar{\Phi}+\bar{\delta}$ are in $\mathcal{L}_{0}$.

The irem 4.1 Assume $J_{j}^{N}$ actiofies (H2), (HS) and (H4). Furthermore, asoume the approximate gradient satiefies conditions (H5) and (H6) and that the update is constructed so that (HY) holds. Then, for a sufficiently fine discretization, the sensitivity equation method produces a sequence of iterates such that

$$
\liminf _{h \rightarrow \infty}\left\|g_{h}\right\|=0 \text {. }
$$

Proof Assume to the contrary that liminf $f_{k \rightarrow \infty}\left\|g_{n}\right\|>0$ and define $\theta_{k}$ such that

$$
\cos \left(\theta_{k}\right)=\frac{\left(-g_{h}, \alpha_{k}\right)}{\left\|g_{h}\right\|\left\|\alpha_{k}\right\|}
$$

and $u_{h} \in \mathcal{Q}$ such that

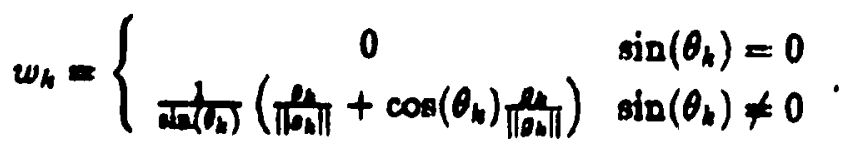

Then $\left(g_{n}, w_{n}\right)=0$ by construction, and

$$
\left\langle g_{k}-\nabla J_{0}^{N}\left(q_{k}\right), w_{k}\right\rangle=-\left\langle\nabla J_{j}^{N}\left(q_{k}\right), w_{k}\right\rangle .
$$


If $\sin \left(\theta_{k}\right) \neq 0$, then $\left\|w_{k}\right\|=1$ and

$$
s_{k}=\left\|s_{k}\right\|\left(-\cos \left(\theta_{k}\right) \frac{g_{k}}{\left\|g_{k}\right\|}+\sin \left(\theta_{k}\right) w_{k}\right)
$$

Let $\mathcal{K}$ denote the set of successful iterations, then

$$
\rho_{k}=\frac{\mathcal{J}_{l}^{N}\left(q_{k}\right)-\mathcal{J}_{l}^{N}\left(q_{k+1}\right)}{\psi_{k}\left(q_{k}\right)-\psi_{k}\left(q_{k+1}\right)}>\eta_{1}
$$

for each $k \in \mathcal{K}$. Lemma 4.1 implies

$$
\mathcal{J}_{g}^{N}\left(q_{k}\right)-\mathcal{J}_{j}^{N}\left(q_{k+1}\right) \geq \frac{\eta_{2} c_{1}}{2}\left\|g_{k}\right\| \min \left\{\delta_{k}, \frac{c_{1}\left\|g_{k}\right\|}{c_{3}}\right\} \text {. }
$$

Since $\mathcal{J}_{j}^{N}$ is bounded below, by (H2), the above condition implies $\lim _{k \rightarrow \infty, k \in K} \delta_{k}=0$. Therefore, as $\delta_{k}$ is decreased in unsuccessful iterations, $\lim _{k \rightarrow \infty} \delta_{k}=0$. We now have the conditions for Lemma 4.2, and

$$
\lim _{h \rightarrow \infty} \frac{\left\langle-g_{h}, s_{k}\right\rangle}{\left\|g_{h}\right\|\left\|s_{k}\right\|}=1 \text {. }
$$

Thus $\lim _{k \rightarrow \infty} \cos \left(\theta_{k}\right)=1$ and $\lim _{k \rightarrow \infty} \sin \left(\theta_{k}\right)=0$.

Consider the expression

$$
1-p_{k}=\frac{\psi_{k}\left(q_{k}\right)-\psi_{k}\left(q_{k+1}\right)-\left(\mathcal{J}_{k}^{N}\left(q_{k}\right)-\mathcal{J}_{g}^{N}\left(q_{k+1}\right)\right)}{\psi_{k}\left(q_{k}\right)-\psi_{k}\left(q_{k+1}\right)}
$$

by Lemma 4.3 and the definition of $\psi_{k}$, we get

$$
1-\rho_{k} \leq \frac{\frac{1}{2}\left(c_{2}+L\right)\left\|s_{k}\right\|^{2}-\left\langle g_{k}-\nabla J_{k}^{N}\left(q_{k}\right), s_{k}\right\rangle}{\left(-g_{k}, s_{k}\right)-\frac{1}{2}\left(H_{k} s_{k}, s_{k}\right)} \text {. }
$$

Using bypothesis (H7),

$$
1-\rho_{k}<\frac{\frac{1}{2}\left(c_{2}+L\right)\left\|s_{k}\right\|^{2}-\left\langle g_{k}-\nabla J_{j}^{N}\left(g_{k}\right), s_{k}\right\rangle}{\left(-g_{k}, s_{k}\right\rangle} \text {. }
$$

Subatituting expression (62) and using $\left\|\alpha_{k}\right\|<\delta_{k}$, we get

$$
1-\rho_{k} \leq \frac{\frac{2}{2}\left(g_{2}+L\right) \delta_{h}-\frac{\cos \left(\varphi_{h}\right)}{\left\|k_{k}\right\|}\left\langle g_{k}-\nabla J_{i}^{N}\left(g_{k}\right), g_{k}\right\rangle-\left\langle g_{k}-\nabla J_{g}^{N}\left(q_{k}\right), w_{h}\right\rangle \sin \left(\theta_{k}\right)}{\left\|g_{k}\right\| \cos \theta_{h}} .
$$

By Lemma 4.4 and the Cauchy-Schwarz inequality, $\left\langle\nabla J_{j}^{N}\left(q_{n}\right), w_{n}\right\rangle$ is bounded and we consider the limit $a k \rightarrow \infty$,

$$
\lim _{h \rightarrow \infty} 1-\rho_{k}=\lim _{k \rightarrow \infty} \frac{\left\langle g_{k}-J_{g}^{N}\left(g_{k}\right), g_{k}\right\rangle}{\left\|g_{k}\right\|^{2}} \leq \frac{\mid g_{k}-J_{j}^{N}\left(q_{k}\right) \|}{\left\|g_{k}\right\|}
$$


Since liminf $k_{k \rightarrow \infty}\left\|g_{k}\right\|>0$ and $g_{k}$ is asymptotically concistent, we can select a suffciently fine discretization such that

$$
\lim _{h \rightarrow \infty} 1-\rho_{k}<1-\eta_{2}
$$

Hence, $\rho_{k}>\eta_{2}$ which implies $\delta_{k+1}>\delta_{k}, 2$ contradiction.

\section{Duct Design Problem}

In this section, we use the duct design problem to illustrate the implementation of the sensitivity equation method. To begin with, we will introduce the discrete approach for finding design sensitivities in order to compare it with the sensitivity equation approach.

\subsection{Discrete Sensitivities}

To obtain an algorithm for the sensitivities $\frac{8}{q} u^{N}(q)=\left\{\frac{g}{g} u_{j}^{N}(q)\right\}_{j=1}^{N}$, the system of nonlinear equations (32) is differentiated, yielding

$$
\frac{F_{j+1 / 2}-F_{j-1 / 2}}{h}+g\left(u_{j}^{N}, \frac{\partial}{\partial q} u_{j}^{N}, A\left(x_{j}\right), \frac{\partial}{\partial q} A\left(x_{j}\right)\right)=0 .
$$

where $F_{j+1 / 2}$ is determizeo by the scheme used to compute the flow. If the EnquistOsher scheme was used,

$$
F_{j+1 / 2}^{E O}= \begin{cases}f_{j+1} & u_{j}^{N}, u_{j+1}^{N} \leq u_{s i} \\ f_{j} & u_{j}^{N}, u_{j+1}^{N} \geq u_{0} \\ 0 & u_{j}^{N}<u_{0}<u_{j+1}^{\prime \prime} \\ f_{j}+f_{j+1} & u_{j+1}^{N}<u_{0}<u_{j}^{N},\end{cases}
$$

or if the artificial viscosity scheme was used,

$$
f_{i+1 / 2}^{A V}=\frac{1}{2}\left(f_{j+1}+f_{j}-\alpha\left(\frac{\partial}{\partial q} u_{j+1}^{N}-\frac{\partial}{\partial q} u_{j}^{N}\right)\right)
$$

where $f_{j}=f\left(u_{j}^{N}, \frac{g}{\delta_{q}} u_{j}^{N}\right)$,

$$
f\left(u, \frac{\partial}{\partial q} u\right)=\left(1-\frac{A}{u^{2}}\right) \frac{\partial}{\partial q} u
$$

and

$$
g\left(u, \frac{\partial}{\partial q} u, A, \frac{\partial}{\partial q} A\right)=\frac{\partial}{\partial q}\left(\frac{1}{A} \frac{\partial}{\partial x} A\right)\left(\bar{\gamma} u-\frac{A}{u}\right)+\left(\frac{1}{A} \frac{\partial}{\partial x} A\right)\left(\bar{\gamma}+\frac{A}{u^{2}}\right) \frac{\partial}{\partial q} u .
$$


This differentiated scheme can now be used to compute $\frac{g}{g} u^{N}$.

\subsection{Sensitivity Equation}

We now present the implementation for the sensitivity equation approach. We begin by differentiating the flow equation (26) with respect to the parameter $q$. Thus

$$
\begin{gathered}
\frac{\partial}{\partial x} f\left(u, \frac{\partial}{\partial q} u\right)+\bar{g}\left(u, \frac{\partial}{\partial q} u, A, \frac{\partial}{\partial q} A\right)=0 \\
\frac{\partial}{\partial q} u(0)=0 \quad \text { and } \quad \frac{\partial}{\partial q} u(1)=0
\end{gathered}
$$

is the sensitivity equation for this problem. Note that the sensitivity equation is a linear equation with variable coefficients (determined by $u$ ). There bas been little analysis of numerical schemes to approximate equations of this type. However, for this two point boundary value problem, the same numerical schemes (Enquist-Osher and artificial viscosity) provide convergent algorithms. As in the approximation of (26), we consider $\left(\frac{g}{B_{q}} u\right)_{j}^{N}$ to be the average censitivity in the $j$ th cell. A system of norlinear equations for $\left(\frac{g}{\partial q} u\right)^{N}(q)=\left\{\left(\frac{g}{\partial q} u\right)_{j}^{N}(q)\right\}_{j=1}^{N}$ can be found by integrating (68) over each cell,

$$
\begin{aligned}
& \frac{f\left(u\left(x_{j}+\frac{h}{2}\right), \frac{\partial}{g_{q}} u\left(x_{j}+\frac{h}{2}\right)\right)-f\left(u\left(x_{j}-\frac{h}{2}\right), \frac{\partial}{\delta_{l}} u\left(x_{j}-\frac{h}{2}\right)\right)}{h} \\
& \quad+\bar{g}\left(u_{j}^{N},\left(\frac{\partial}{\partial q} u\right)_{j}^{N}, A\left(x_{j}\right), \frac{\partial}{\partial q} A\left(x_{j}\right)\right)=0,
\end{aligned}
$$

$j=1, \ldots, N$, where we assume $A$ and $\frac{8}{g} A$ are nearly constant over each cell. As before, the terms $f\left(u\left(x_{j}+\frac{h}{2}\right), \frac{g_{i}}{f_{i}} u\left(x_{j}+\frac{h}{2}\right)\right)$ are replaced by the cell center values $f_{j}$ and $f_{j+1}$. Uaing the Enquist-Osher scheme, we obtain

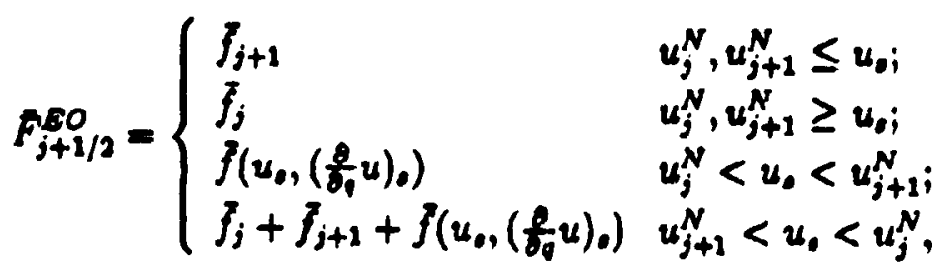

and obtain

$$
F_{j+1 / 2}^{A V}=\frac{1}{2}\left(f_{j+1 / 2}+f_{j}-\alpha\left(\left(\frac{\partial}{\partial q} u\right)_{j+1}^{N}-\left(\frac{\partial}{\partial q} u\right)_{j}^{N}\right)\right)
$$


for the artificial viscosity scheme. It is obvious that the approximation of the sensitivity equations depends on the approximation of the flow equations. As described earlier, we use the notation $\left(\frac{\partial}{\partial_{q}} u\right)^{N, M}$ to represent using scheme $N$ to approximate the flow equatior and scheme $M$ to approximate the sensitivity equation.

\subsection{Convergence Results}

The convergence resulk provided ir Theorem 4.1 can be proved for the case when the artificial viscosity scieme is used to approximate the flow and the Enquist-Osher scheme is used to approximate the sensitivities in Algorithm 3.1. For this problem, we assume (the (H1) in Theorem 4.1) that

$$
\mathcal{Q}=\left[A_{\mathrm{ln}}, A_{\text {out }}\right], \quad \mathcal{Q}_{0}=\left(A_{\text {ln }}, A_{\text {out }}\right),
$$

and

$$
\mathcal{C}_{0}=\left\{q \in \mathcal{Q} \mid \mathcal{J}_{g}^{N_{A V}}(q) \leq \mathcal{J}_{g}^{N_{A V}}\left(q_{0}\right)\right\} .
$$

The objective function $\mathcal{J}_{0}^{N_{A V}}$ given above is obviously bounded below (by zero if all of the quadrature weights are nonnegative) satiffying (H2). The hypothesis (H3), the differentiability of

$$
J_{a}^{N_{\Delta v}}(q)=\sum_{i=1}^{\ell} c_{i}\left(u^{N_{A v}}\left(x_{i}, q\right)-\hat{u}\left(x_{i}\right)\right)^{2}
$$

on $\mathcal{Q}_{0}$ and hypothesis (H4), the Lipschitz cuntinuity of the derivative, follow from the following

Lemma 5.1 The approximate solution $u^{N_{A V}}$ is differentiable and the derivative is Lipschitz continuous on $\boldsymbol{Q}_{0}$.

Proof The approximate wolution, $u^{N_{A V}}$ is the root of the ponlinear equations

$$
W\left(u^{N_{A V}}, q\right)=\left[F_{j+1 / 2}^{A V}\left(u^{N_{A V}}, q\right)-F_{j-1 / 2}^{A V}\left(u^{N_{A V}}, q\right)\right]+g_{j}\left(u^{N_{A V}}, q\right)=0,
$$

where $F_{j+1 / 2}^{A V}$ and $g$ are $C^{\infty}$ functions of their arguments (for $u^{N_{A V}}>0$ ). Then by the implicit function theorem, the map

$$
q \rightarrow u^{N_{A V}}(q)
$$

is Lipschitz continuously differentiable.

We point out that the differentiability of the approximate objective functional is strongly dependent on the discretization scheme used in the approximation. For 
example, the objective functional associated with a Gidunov approximation of the flow is not differentiable, a result of matching a parameter dependent discontinuity on a discrete set of points [4]. Finding feasible optimination strategies for this problem has been the focus of recent work, see e.g. [4], [19] and [23]. However, for the purpose of this discussion, the artificial viscosity scheme provides a smootb enough approximate objective fuaction.

The hypothesis (H5) is guaranteed (for some discretization level) by the asymptotic consistency shown below.

Theorem 5.1 For the one dimensional Euler equations, the derivative $\left(\frac{g}{g_{q}} \mathcal{J}\right)_{g}^{N_{A V}, M_{s 0}}$, where the flow is approximated using the artificial viscosity approximation and the sensitivities are approximated using the Enguist-Osher scheme, is asymptotically consistent to $\frac{8}{8 Q} \mathcal{J}_{g}^{N_{A v}}$.

Proof Consider the norm used in the definition of asymptotic consistency:

$$
\begin{aligned}
& \left|\frac{\partial}{\partial q} J_{g}^{N_{A V}}-\left(\frac{\partial}{\partial q} \mathcal{J}\right)_{g}^{N_{A V}, M_{D O}}\right| \leq\left|\frac{\partial}{\partial q} J_{g}^{N_{A V}}-\left(\frac{\partial}{\partial q} \mathcal{J}\right)^{N_{A V}, M_{A V}}\right| \\
& +\left|\left(\frac{\partial}{\partial q} \mathcal{J}\right)^{N_{A V}, N_{A V}}-\left(\frac{\partial}{\partial q} \mathcal{J}\right)^{N_{A V}, M_{x I}}\right| \\
& +\left|\left(\frac{\partial}{\partial q} \mathcal{J}\right)_{g}^{N_{A V}, M_{B},}-\left(\frac{\partial}{\partial q} \mathcal{J}\right)_{0}^{N_{A V}, M_{\Sigma 0}}\right| \text {. }
\end{aligned}
$$

The first term on the right hand side vanishes since using the artificial viscosity scheme for approximating both the flow and sensitivity equations leads to consistent derivatives. The 'ast two terms go to zero as the approximations $N_{A V}, M_{A V}$ and $M_{E O}$ are refined, since the artificial viscority and Enquist-Osher achemes converge when used to approximate the sensitivity equation, $\left(\frac{f}{g}, u\right)^{N_{A V}, M_{\Sigma 0}}$ is the exact solution to the sensitivity equation given $u^{N_{A V}}$.

The hypothesis (H6) can be enforced by the optimization algorithm by rejecting steps which violate this condition and shrinking the trust-region radius. This procedure eventually creates a step which satisfies (H6), since the limit of ihis procedure would produce a step in the steepeat descent direction.

Finally, (H7) can be enforced by the secant update atrategy. Therefore, we have shown that these approximation echernes axtisfy the conditions of Theorem 4.1. Nume:ical computations using these sensitivity schemes are provided below. 


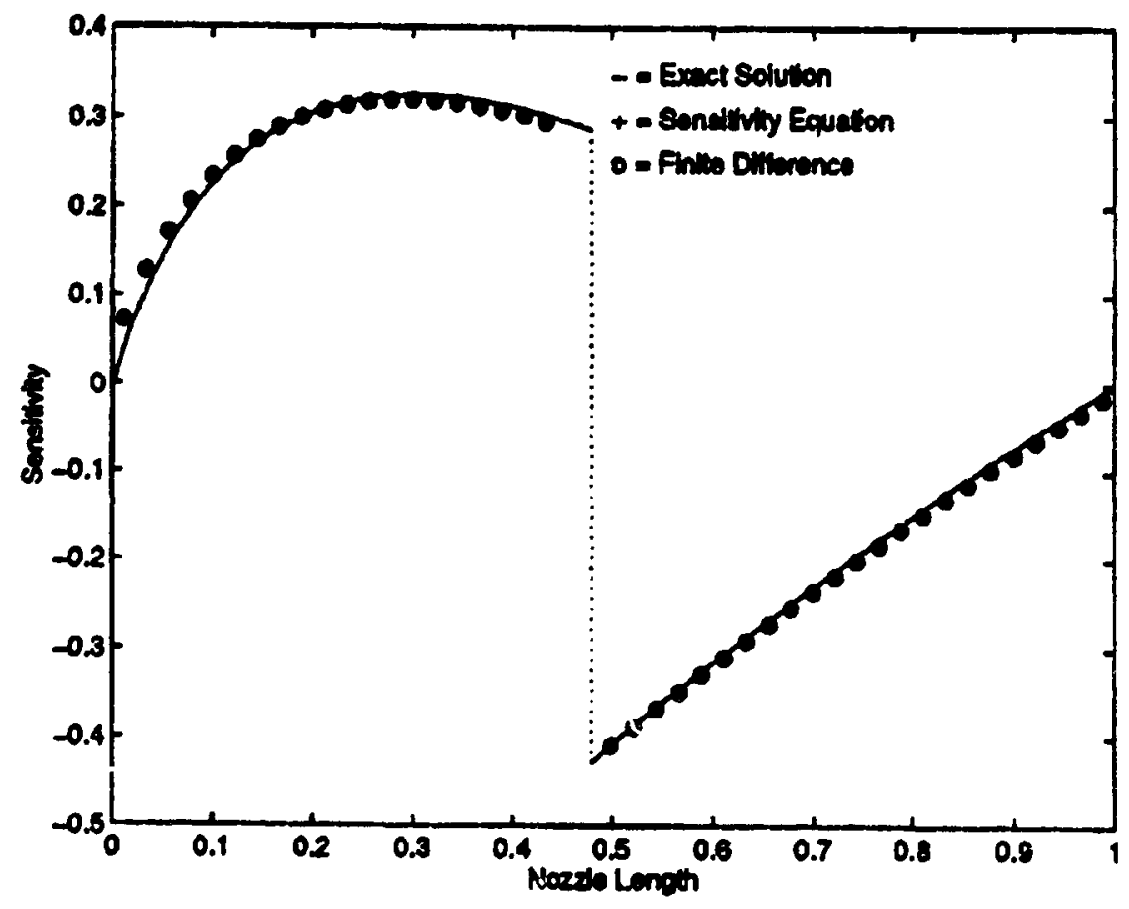

Figure 3: Design Sensitivity Approximations Using Enquirt-Onher Scheme

\subsection{Numerical Results}

The sensitivity of the velocity with respect to the Bezier parameter, $q$, is presented using the numerical schemes described above. For this computation, the crose-sectional area corresponds to an element of $B$ (see (35)) with $q=1.37125$. The interval $[0,1]$ is divided into 45 cells. In Figure 3, the sensitivity solution using the Enquist-Osher scheme to compute both the flow $u^{N_{50}}$ and the sensitivity $\left(\frac{f_{0}}{g_{1}}\right)^{N_{n 0}, N_{00}}$ is compared with the closed form sensitivity solution. In addition, the sensitivities computer via finite differences of Enquist-Osher solutions using a finite difference step size of $\Delta q=\left(1 \times 10^{-6}\right) q$ are also provided. Excellent agreement is seen for both of these methods. The only discrepancy is in the cell to the left of the shock, where numerical discripation appeare in the flow solution.

The corresponding design sensitivities which are computed using only the artificial viscosity schemes are shown in Figur 4. As above, the agreement is excellent except where dissipation errors appear in the flow approximations. In this case, these erron appear over more celle near the shock.

Note that the computation of these rensitivities were performed efficiently, rela. 


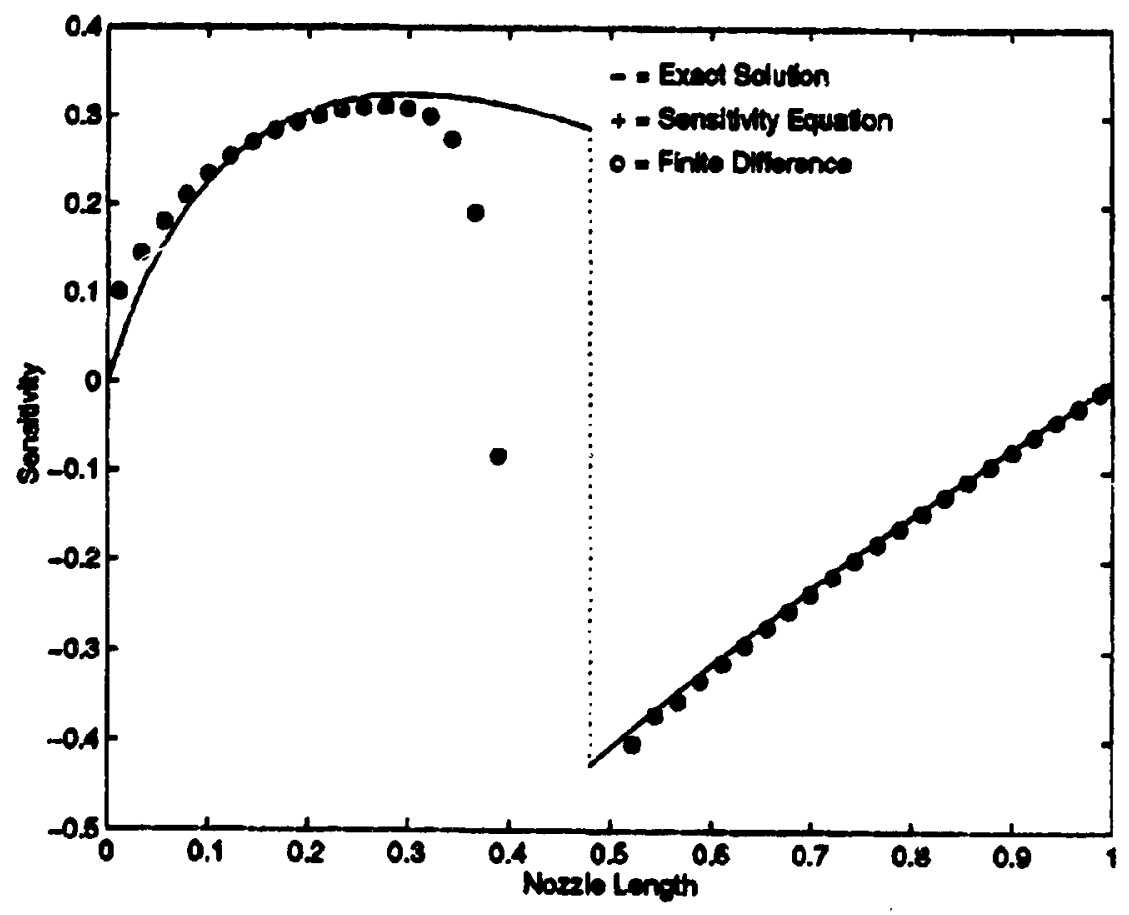

Figure 4: Deaign Senvitivity Approximation Uaing Artificial Viscosity Scheme

tive to the cost of a flow apprcximation. The flow approximation requires solving a system of nonlinear equations. The susitivity approximation, on the other hand, only requires solving a linear system since the sensitivity appears only linearly in the definition of $\bar{f}$ and $\bar{g}$. Moreover, if the Newton method is used to solve the nonlinear system, then the linear system is already available in factored form. Therefore, the sensitivities can be computed using less computational time than required for one Newton step. Computational efficiencies such a this can be missed if the fiow algorithm is simply differentiated.

Note that as long as $\left(\frac{g}{g} u\right)$, is bounded,

$$
f\left(u_{e},\left(\frac{\partial}{\partial q} u\right)\right)=\left(1-\frac{F}{u_{q}^{2}}\right)\left(\frac{\partial}{\partial q} u\right)=0,
$$

since $\bar{H}=u_{*}^{2}$. Thus, one observes that the numerical algorithens to compute either $\frac{g}{b q} u^{N_{z 0}}$ or $\left(\frac{g}{t} u\right)^{N_{10}, M_{x 0}}$ are equivalent. This leads to the fact that using the Enquist-Osber scheme to approximate botb the flow and sensitivity equations produces consistent gradients. In addition, it is exoily seen that using the artificial viacosity scheme to approximate both equations also produces consistent gradients. 
Table 1: A Comparieon of Gradiente at the Optimum for Various Meab Sizes

\begin{tabular}{|c|c|c|c|}
\hline $\mathbf{N}$ & 9. & $J^{N_{A V}}$ & 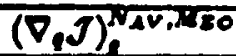 \\
\hline 16 & 1.3538 & 0.011707 & -0.056521 \\
\hline 48 & 1.3437 & 0.004800 & -0.001566 \\
\hline 135 & 1.3526 & 0.002486 & -0.000012 \\
\hline 225 & 1.3643 & 0.002476 & 0.007602 \\
\hline 315 & 1.3563 & 0.002646 & 0.026731 \\
\hline 405 & 1.3554 & 0.002816 & 0.001584 \\
\hline
\end{tabular}

However, if the artificial viscosity scheme is used to approximate the flow and the Enquist-Osher scheme is used to approximate the sensitivity equations, the gradients are not consistent but asymptotically consistent.

Numerical results for this asymptotically consistent case are provided in Table I.

\section{Forebcdy Simulator Design Problem}

We now describe the implementation of the sensitivity equation method for the forebody simulator design problem described in Section 2 . As in the duct design problem, we begin by presenting the equations which comprise the discrete sensitivity scheme in order to compare and contrast the two methods. Unlike the duct problem, we have no theoretical convergence reoults for the FBS desigo problem. However, the numerical experiments below show that the SEM still converges.

\subsection{Discrete Senuitivities}

Differentiating the numerical scheme (16) with respect to a design parameter, represented by $q$, leads to the following scheme:

$$
\begin{aligned}
& {\left[I+\Delta t \delta_{\ell} \bar{A}^{n}-\nabla_{\ell}\left(\Psi_{\ell}^{(2)}+\Psi_{\ell}^{(1)}\right) \Delta_{\ell} J_{M}\right] \times} \\
& {\left[I+\Delta t \delta_{\eta} B^{n}-\nabla_{\eta}\left(\Psi_{\eta}^{(2)}+\Psi_{\eta}^{(4)}\right) \Delta_{\eta} J_{M}\right] \Delta \frac{\partial}{\partial q} Q^{n}=} \\
& -\left[\Delta t \delta_{\xi} \frac{\partial}{\partial q} \bar{A}^{n}-\nabla_{\ell}\left(\frac{\partial}{\partial q} \psi_{\ell}^{(2)}+\frac{\partial}{\partial q} \Psi_{l}^{(1)}\right) \Delta_{\zeta} J_{M}-\nabla_{\ell}\left(\psi_{\ell}^{(2)}+\psi_{\ell}^{(1)}\right) \Delta_{\zeta} \frac{\partial}{\partial q} J_{M}\right] \times \\
& {\left[I+\Delta t \delta_{\eta} B^{n}-\nabla_{\eta}\left(\Psi_{\eta}^{(2)}+\Psi_{\eta}^{(4)}\right) \Delta_{\eta} J_{M}\right] \Delta Q^{n}} \\
& -\left[I+\Delta t \delta_{l} A^{n}-\nabla_{l}\left(\nabla_{l}^{(2)}+\Psi_{l}^{(4)}\right) \Delta_{l} J_{M}\right] \times \\
& {\left[\Delta t \delta_{\eta} \frac{\partial}{\partial q} B^{n}-\nabla_{\eta}\left(\frac{\partial}{\partial q} \psi_{\eta}^{(2)}+\frac{\partial}{\partial q} \psi_{\eta}^{(4)}\right) \Delta_{\eta} J_{M}-\nabla_{\eta}\left(\psi_{\eta}^{(2)}+\psi_{\eta}^{(4)}\right) \Delta_{\eta} \frac{\partial}{\partial q} J_{M}\right] \Delta \dot{Q}^{n}}
\end{aligned}
$$




$$
\begin{aligned}
& -\Delta t \delta_{t} \frac{\partial}{\partial q} F^{n}-\Delta t \delta_{n} \frac{\partial}{\partial q} G^{n} \\
& +\Delta t \nabla_{l}\left(\frac{\partial}{\partial q} \Psi_{l}^{(2)}-\frac{\partial}{\partial q} \Psi_{l}^{(4)} \Delta_{\ell} \nabla_{l}\right) \Delta_{l}\left(J_{M} Q^{n}\right) \\
& +\Delta t \nabla_{\xi}\left(\Psi_{\xi}^{(2)}-\Psi_{\xi}^{(4)} \Delta_{\xi} \nabla_{\xi}\right) \Delta_{\ell} \frac{\partial}{\partial q}\left(J_{M} Q^{n}\right) \\
& +\Delta t \nabla_{\eta}\left(\frac{\partial}{\partial q} \Psi_{\eta}^{(2)}-\frac{\partial}{\partial q} \Psi_{\eta}^{(4)} \Delta_{\eta} \nabla_{\eta}\right) \Delta_{\eta}\left(J_{M} \delta^{n}\right) \\
& +\Delta t \nabla_{\eta}\left(\Psi_{\eta}^{(2)}-\Psi_{\eta}^{(1)} \Delta_{\eta} \nabla_{\eta}\right) \Delta_{\eta} \frac{\partial}{\partial q}\left(J_{M} Q^{n}\right) \text {. }
\end{aligned}
$$

The equation representing the boundary conditions are also differentiated. Note that the above sensitivity echeme requires derivatives of the mapping, $\frac{8}{\gamma_{1}} \mathcal{M}$ (denoted as mesh sensitivities) and the diesipation terms, $\frac{8}{\delta_{q}} \Psi^{(2)}$ and $\frac{g}{g_{q}} \Psi^{(4)}$. Evaluation of $\frac{\partial}{\partial q} \mathcal{M}$ is given by differentiating the scheme which determines $\mathcal{M}$, see e.g. [20]. Other methods for approximating $\frac{9}{y_{q}} \mathcal{M}$ have aleo been investigated, see e.g. [25]. We see from (74) that terms containing these expressions represent a significant portion of the computational effort, aside from the fact that $\frac{8}{q_{q}} \mathcal{M}, \frac{\theta_{q}}{q_{q}} \Psi^{(2)}$ and $\frac{8}{\delta_{q}} \Psi^{(4)}$ themselves nead to be determined.

\subsection{Sensitivity Equation}

The senoitivity equation approach to computing design sensitivities is presented below. To begin with, we differentiate the Euler equations and aesociated boundary conditions with reapect to the design parameter $q$, which leads to:

$$
\frac{\partial F_{9}}{\partial x}+\frac{\partial G_{q}}{\partial y}=0
$$

where

$$
\begin{aligned}
& F_{q}=\frac{\partial}{\partial q} u Q+u Q_{q}+\left[\begin{array}{c}
0 \\
\frac{\theta}{\delta_{q}} P \\
0 \\
\delta_{q} P u+P g_{q} u
\end{array}\right] \text {, } \\
& G_{q}=\frac{\partial}{\partial q} v Q+v Q_{q}+\left[\begin{array}{c}
0 \\
0 \\
f_{q} P \\
g_{q} P v+P \frac{g_{q}}{\delta_{q}}
\end{array}\right], \quad Q_{1}=\left[\begin{array}{c}
\delta_{q} \rho \\
f_{q}(\rho u) \\
f_{q}(\rho v) \\
\frac{g}{\delta_{q}} E
\end{array}\right] \text {, }
\end{aligned}
$$


and where

$$
\frac{\partial}{\partial q} u=\left[\frac{\partial}{\partial q}(\rho u)-\frac{\partial}{\partial q} \rho \frac{\rho u}{\rho}\right] / \rho \text { and } \frac{\partial}{\partial q} v=\left[\frac{\partial}{\partial q}(\rho v)-\frac{\partial}{\partial q} \rho \frac{\rho v}{\rho}\right] / \rho,
$$

since $\rho \neq 0$.

We are now free to apply any appropriate scheme to solve (75). In particular, it is possible to use a method which takes advantage of the linearity of the sensitivity equation. However, in this work, the same scheme used to solve the flow equations is used to approximate the sensitivity equations, which leads to an efficient computational scheme as in the discrete approach [2]. This scheme is described below.

This equation may now be transformed to generalized coordinates, so that the finite differencing can be done more easily. It makes sense to use the same transformation (which is equivalent to using the same mesh) that was used in the solution of the Euler equations. Thus the resulting system is

$$
\frac{\partial \bar{F}_{q}}{\partial \xi}+\frac{\partial \bar{G}_{q}}{\partial \eta}=0
$$

where

$$
\begin{aligned}
& F_{q}=U Q_{q}+U_{q} \bar{Q}+\frac{\partial}{\partial q} P J_{M}^{-1}\left[\begin{array}{c}
0 \\
\frac{\partial}{\partial q} \\
\frac{\alpha}{\partial y} \\
U
\end{array}\right]+P J_{M}^{-1}\left[\begin{array}{c}
0 \\
0 \\
0 \\
U_{q}
\end{array}\right], \\
& G_{q}=V Q_{q}+V_{q} Q+\frac{\partial}{\partial q} P J_{M}^{-1}\left[\begin{array}{c}
0 \\
\frac{\partial_{\eta}}{\partial q} \\
\frac{\partial_{q}}{\partial_{q}} \\
V
\end{array}\right]+P J_{M}^{-1}\left[\begin{array}{c}
0 \\
0 \\
0 \\
V_{q}
\end{array}\right],
\end{aligned}
$$

where

$$
U=\nabla \xi \cdot(u, v) \text { and } U_{q}=\nabla \xi \cdot\left(\frac{\partial}{\partial q} u, \frac{\partial}{\partial q} v\right) \text {, }
$$

and

It can be shown that

$$
V=\nabla \eta \cdot(u, v) \text { and } V_{1}=\nabla \eta \cdot\left(\frac{\partial}{\partial q} u, \frac{\partial}{\partial q} v\right)
$$

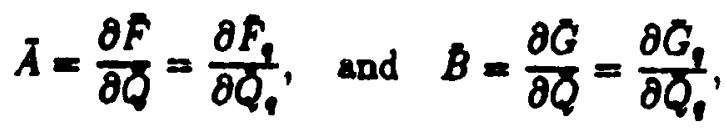


80 that the discretization has the same factored form as the Eulex equations, thus

$$
\begin{aligned}
& {\left[I+\Delta t \delta_{\xi} \bar{A}^{n}-\nabla_{\zeta}\left(\Psi_{\xi}^{(2)}+\Psi_{\xi}^{(1)}\right) \Delta_{\zeta} J_{M}\right] \times} \\
& {\left[I+\Delta t \delta_{\eta} B^{n}-\nabla_{\eta}\left(\Psi_{\eta}^{(2)}+\Psi_{\eta}^{(1)}\right) \Delta_{\eta} J_{M}\right] \Delta\left(\frac{\partial}{\partial q} \bar{Q}\right)^{n}=} \\
& -\Delta t \delta_{q}\left(\tilde{F}_{q}\right)^{n}-\Delta t \delta_{\eta}\left(\boldsymbol{G}_{q}\right)^{n} \\
& +\Delta t \nabla_{\ell}\left(\Psi_{\zeta}^{(2)}-\Psi_{\ell}^{(4)} \Delta_{l} \nabla_{\ell}\right) \Delta_{\zeta}\left(J_{M} Q\right)^{n} \\
& +\Delta t \nabla_{\eta}\left(\Psi_{\eta}^{(2)}-\Psi_{\eta}^{(1)} \Delta_{\eta} \nabla_{\eta}\right) \Delta_{\eta}\left(J_{M} Q\right)^{n}
\end{aligned}
$$

Since the left hand side matrices are the same, a right hand side vector needs to be formed for each design rensitivity. In addition, the boundary condition type is the same for both the Euler and sensitivity equations. The boundary conditions are determined using implicit differentiation.

Note that this scheme is similar to the diecrete sensitivity approach. However, since the approximation is applied after the differentiation, there are no mesh sensitivity or dissipation sensitivity terms. The other obvious difference is that the boundary condition on the parameter dependent boundary is different.

\subsection{Boundary Conditions}

The boundary conditions for the mensitivity oquation (75) are provided below for the case where the forebody simulator is deacribed by a two parameter Bexies curve (18)(20). Extensions to other forebody descriptione will be obvious. The appropriate conditions are obtained by differentiating the corresponding boundary conditions for the Euler equations. For example, at the inlet, tie fow $Q_{\text {m }}$ is prescribed and will not vary as the forebody parameters $q=\left(q^{1}, q^{2}\right)$ are changed, thus

$$
Q_{1}=0
$$

at the teat cell inflow. The walls are treated in a similar fachion. However, the boundary condition at the forebody simulator surface requires more attention. This is because the points where the condition is evaluated are parameter dependent.

We study the treatment of condition (5) in detail. The normal vector to the forebody surface is

$$
\hat{n}=\left(-\frac{\partial}{\partial s} \Gamma_{y}(s ; q), \frac{\partial}{\partial s} \Gamma_{s}(s)\right) \sqrt{\left(\frac{\partial}{\partial s} \Gamma_{s}(s)\right)^{2}+\left(\frac{\partial}{\partial s} \Gamma_{y}(s ; q)\right)^{2}} .
$$


Thus, the boundary condition (5) can be written as

$$
-u\left(\Gamma_{s}(s), \Gamma_{y}(s ; q) ; q\right) \frac{\partial}{\partial s} \Gamma_{y}(s ; q)+v\left(\Gamma_{s}(s), \Gamma_{y}(s ; q) ; q\right) \frac{\partial}{\partial s} \Gamma_{s}(s)=0 .
$$

The correoponding rensitivity equation boundary condition for the first parameter, $q^{2}$, can be obtained via differentiation, i.e.,

$$
\begin{aligned}
-\frac{\partial}{\partial q^{2}} u\left(\Gamma_{s}(s), \Gamma_{y}(s ; q) ; q\right) & \frac{\partial}{\partial s} \Gamma_{y}(s ; q)+\frac{\partial}{\partial q^{2}} v\left(\Gamma_{y}(s ; q), \Gamma_{z}(s) ; q\right) \frac{\partial}{\partial s} \Gamma_{s}(s)= \\
& \frac{\partial}{\partial y} u\left(\Gamma_{z}(s), \Gamma_{y}(s ; q) ; q^{\prime} ; \frac{\partial}{\partial q^{1}} \Gamma_{y}(s ; q) \frac{\partial}{\partial s} \Gamma_{y}(s ; q)\right. \\
& +u\left(\Gamma_{x}(s), \Gamma_{y}(s ; q) ; q\right) \frac{\partial^{2}}{\partial s \partial q^{2}} \Gamma_{y}(s ; q) \\
& -\frac{\partial}{\partial y} v\left(\Gamma_{x}(s), \Gamma_{y}(s ; q) ; q\right) \frac{\partial}{\partial q^{2}} \Gamma_{y}(s ; q) \frac{\partial}{\partial s} \Gamma_{x}(s) .
\end{aligned}
$$

This is simply a nonhomogeneous version of condition (5), namely,

$$
\left(\frac{\partial}{\partial q^{2}} u, \frac{\partial}{\partial q^{2}} v\right) \cdot \hat{n}=\frac{\partial}{\partial y} u \frac{\partial}{\partial q^{2}} \Gamma_{y} \frac{\partial}{\partial s} \Gamma_{y}+u \frac{\partial^{2}}{\partial s \partial q^{2}} \Gamma_{y}-\frac{\partial}{\partial y} v \frac{\partial}{\partial q^{2}} \Gamma_{y} \frac{\partial}{\partial s} \Gamma_{z}
$$

Using the same techniques, the boundary conditions corresponding to $(6)$ are:

$$
\begin{aligned}
& \frac{\partial}{\partial n}\left(\frac{\partial}{\partial q^{1}} u_{t}\right)=\frac{\partial^{2}}{\partial x \partial y} u_{t} \frac{\partial}{\partial q} \Gamma_{y} \frac{\partial}{\partial s} \Gamma_{y}+\frac{\partial}{\partial x} u_{t} \frac{\partial^{2}}{\partial s \partial q^{1}} \Gamma_{y}-\frac{\partial^{2}}{\partial y^{2}} u_{t} \frac{\partial}{\partial q^{1}} \Gamma_{y} \frac{\partial}{\partial s} \Gamma_{s}, \\
& \frac{\partial}{\partial n}\left(\frac{\partial}{\partial q^{1}} v_{t}\right)=\frac{\partial^{2}}{\partial x \partial y} v_{t} \frac{\partial}{\partial q} \Gamma_{y} \frac{\partial}{\partial s} \Gamma_{y}+\frac{\partial}{\partial x} v_{t} \frac{\partial^{2}}{\partial s \partial q^{1}} \Gamma_{y}-\frac{\partial^{2}}{\partial y^{2}} v_{t} \frac{\partial}{\partial q^{1}} \Gamma_{y} \frac{\partial}{\partial s} \Gamma_{z} .
\end{aligned}
$$

The analogous boundary conditions for $q^{2}$ are obvious.

\subsection{Numerical Result.}

The sensitivity equation approach, which compute deaigs senoitivities for the two dimensional Eules equation is illustrated below. In this implementation, a right hand side vector for each deaign sensitivity is formed along with the corresponding vector for the flow approximations. The updates for the flow and senaitivity variables are obtained aimultaneously, exploiting the fact that the left hand side matrices are the same.

The desigo ranitivities with respect to the firat Bezier parameter $q^{2}$ were computed for a forebody described by the curve

$$
f=(t(s), \hat{v}(s)), \quad, \in[0,1],
$$


where

$$
\begin{aligned}
& \hat{x}(s)=0.0 B_{0,8}(s)+0.1 B_{1,3}(s)+0.55 B_{2,3}(s)+1.0 B_{3,3}(s), \\
& \hat{y}(s)=\Gamma_{s} B_{0,8}(s)+q^{1} B_{1,3}(s)+q^{2} B_{2,3}(s)+\Gamma_{6} B_{3,3}(s),
\end{aligned}
$$

$q^{1}=0.1, q^{2}=0.15, \Gamma_{a}=0$ and $\Gamma_{b}=0.2$. This curve is twice as long in the $x$-direction as the admissible forebody simulators given in $B$ (cee (18)). Under a uniform inlet flow profile described by the inlet Mach number, $M_{*}=2.0$, the approximate flow variables and sensitivities are computed on a $43 \times 49$ mesh. The sensitivity of the $x$-component of momentum with respect to the Bexier parameter $q^{1}$, computed using the sensitivity equation approach and the finite difference approach (for 4 different step sizes) are plotted along the outflow plane in Figure 5 . The corresponding plots for the Energy sensitivity are provided in Figure 6. Observe that the step size of 0.00001 produces noisy rensitivity values close to the forebody (presumably due to round-off errors). A larger step size of 0.01 gives the best results (when compared to the sensitivity equation approach) near the abock location. The best qualitative behavior appears when the atep size is 0.001 . These figures demonstrate the difficulty of obtaining a satiffactory step size at all resolution levels in the flow domain.

A model forobody simulator design problem is discussed below. To begin witk, we seak the optimum value of the inlot Mach number and two Bezier parameters $\left(\left(q^{1}, q^{2}\right)\right.$, describing a shortened forebody simulator in the admissible et $\left.B\right)$ which minimize the approximate cont functional $\mathcal{J}_{\rho}^{N}$ (given in equation (25)). The flow data $Q$ to be matched is given by the fow $Q^{N}$ corresponding to the forebody shape $f$ deacribed above. We point out that the artificial diasipation in the flow solver produce a "omearing" effect on the fow variables. Therefore, baced on the results for the duct design problem, we expect a rufficiently smooth approximate cont functions. Furthermore, the comparieon of the censitivities in Figures 5 and 6 lead us to believe that the ensitivity equation appraech may produce asymptotically conoistent derivatives.

The sensitivity equation method was applied to the FBS design problem with initial values of the parameters: $M_{a}=2.0, q^{1}=0.10$ and $q^{2}=0.15$. These parameters correapond to thowe used to generate $Q$ (even though that forebody is longer). We present the iteration history in Table II. Observe that there is a drastic reduction in the approximate cost functional in the firnt three iterations. The iteration bistory for the x-component of moment um is given in Figure 7. Note that the front end of 

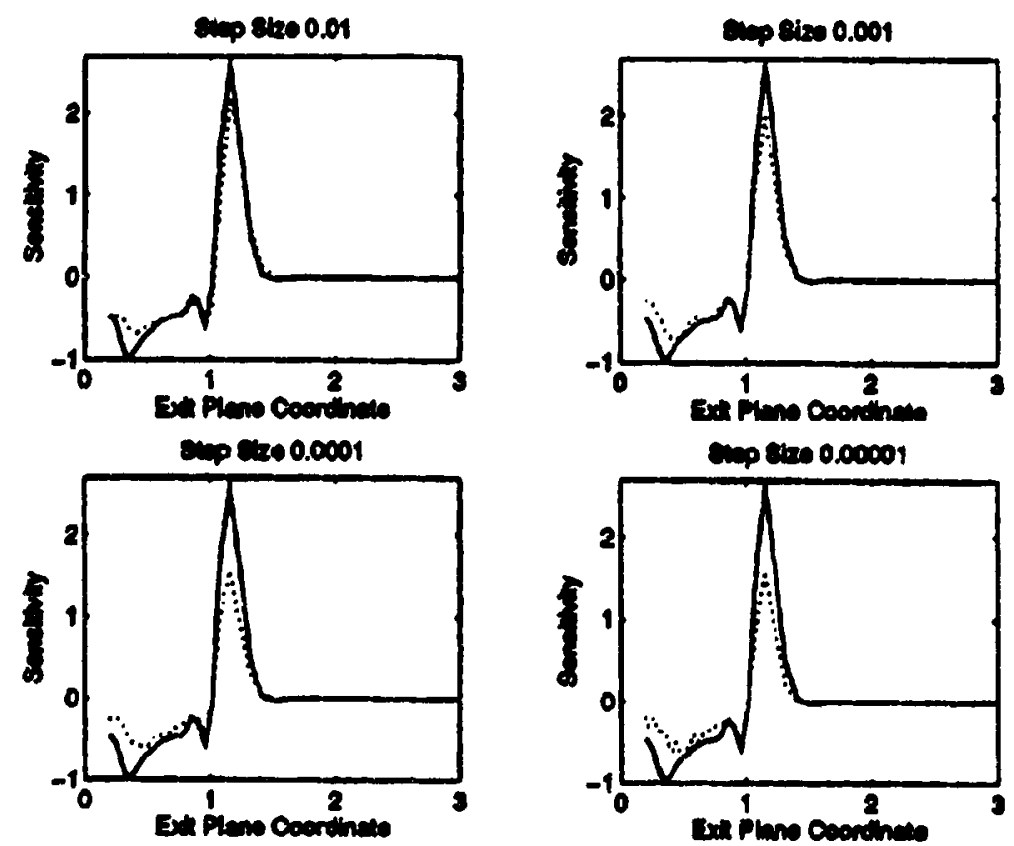

Figure 5: Comparinon of Sensitivities at Outflow: $x$-Component of Momentum
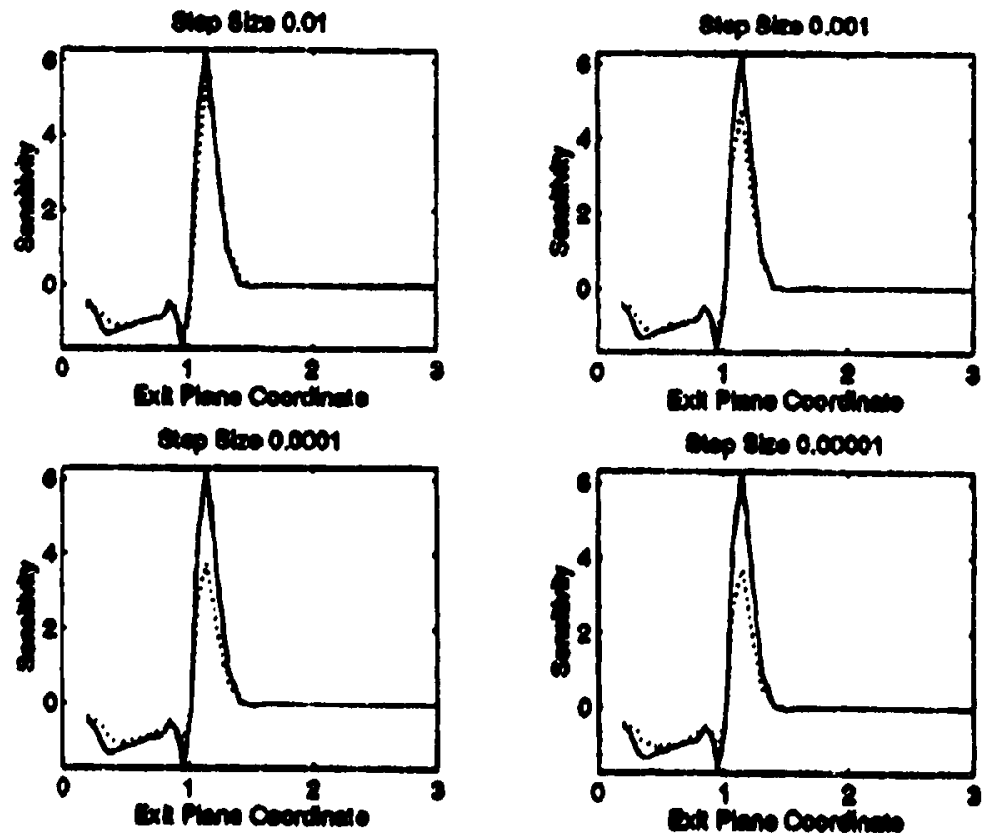

Figure 6: Comparices of Sendtivition at Outeow: Energy 
Table ll: Shortaned Forebody Optimisation

\begin{tabular}{|c|c|c|c|c|c|}
\hline Ileration & $M_{e}$ & 91 & 12 & Cost runetional & Gradient \\
\hline 0 & 2.00000 & 0.10000 & 0.15000 & 3.2336 & 27.1263 \\
\hline 1 & 2.00108 & 0.14608 & 0.17177 & 1.6000 & 11.6285 \\
\hline 2 & 2.01054 & 0.26846 & 0.14162 & 0.3382 & 3.7855 \\
\hline 3 & 2.00897 & 0.30706 & 0.18671 & 0.2334 & 0.4621 \\
\hline 4 & 2.01027 & 0.30139 & 0.14007 & 0.2806 & 0.8808 \\
\hline 8 & 2.01307 & 0.29367 & 0.14787 & 0.2280 & 0.0861 \\
\hline 6 & 2.01670 & 0.28881 & 0.18604 & 0.2271 & 0.6009 \\
\hline 7 & 2.01960 & 0.29011 & 0.16021 & 0.2240 & 0.1613 \\
\hline 8 & 2.01940 & 0.29278 & 0.15821 & 0.2237 & 0.0576 \\
\hline 9 & 2.01936 & 0.29420 & 0.15660 & 0.2232 & 0.0571 \\
\hline 10 & 2.01962 & 0.29489 & 0.16604 & 0.2230 & 0.0275 \\
\hline 11 & 2.01904 & 0.28417 & 0.15608 & 0.2220 & 0.0173 \\
\hline 12 & 2.02000 & 0.29415 & 0.15609 & 0.2220 & 0.0158 \\
\hline
\end{tabular}

the forebody simulator becomes more blunt during the firat two iterations in which a stagnation region is set up in front of the FBS. This bas the effect of moving the shock forward, which comes close to the ahock location created by the long forebody. The remaining iterations are uned to "fine tune" the colution near the FBS. The comparison of the optimal forebody simulator to the flow generated by the long forebody is displeyed in Figure 8. Notice that the ahock location is the same in both flows.

In the optimization above, the initial Heasian was computed using forward differences. This adds some initial expense in the hope for fewer iterations. However, without this technique, using the identity matrix $2 s$ the initial Heasian, the iteration converged in fifteen iterations. Therefore, neither technique showed an advantage.

\subsection{Conclusions}

While no rigorous proof of asymptotically consintent gradients hes been shown for Euler equations, numerical evidence in [3] suggeots that the gradiente may indeed be anymptotically consistent. Similar numerjeal evidence exists for finite dement approximations of the Navier-Stoken equations [b]. 


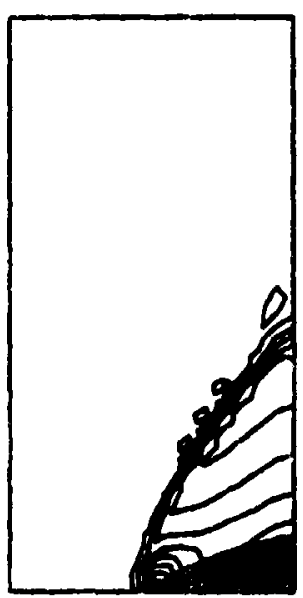

Ireration 0

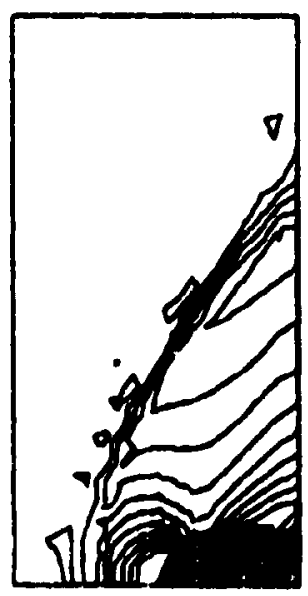

Irenation 4

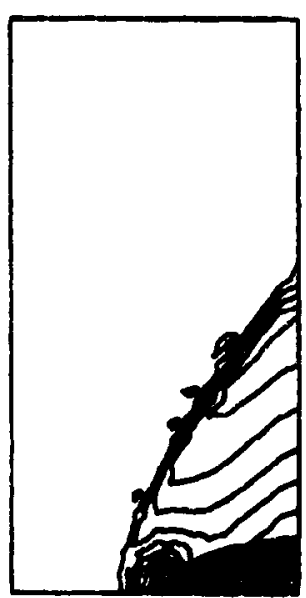

Iteration 1

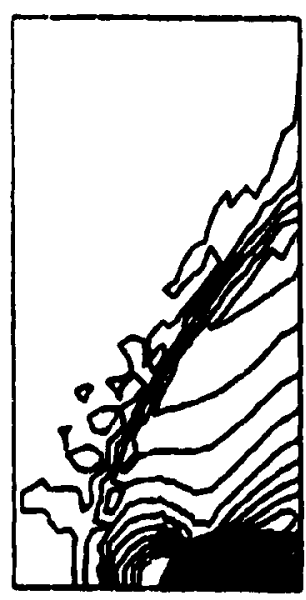

Iteration 6

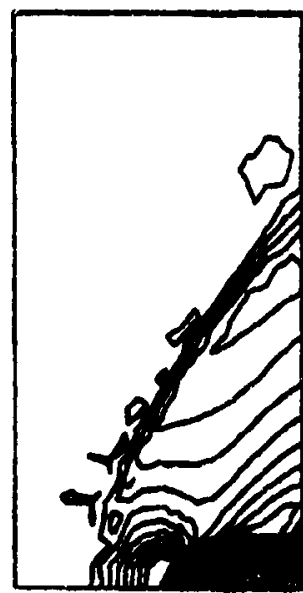

Iteration 2

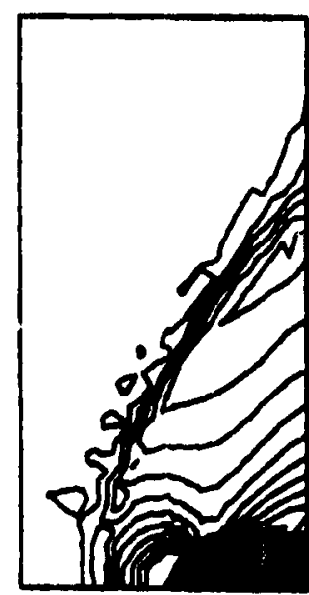

Iteration 8

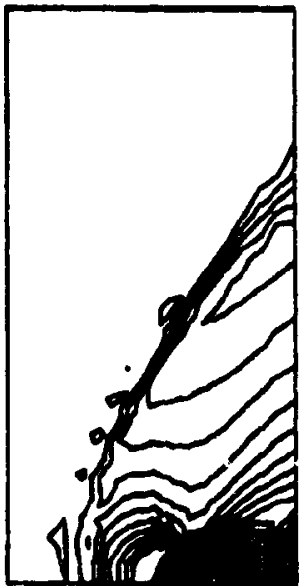

Iteration 3

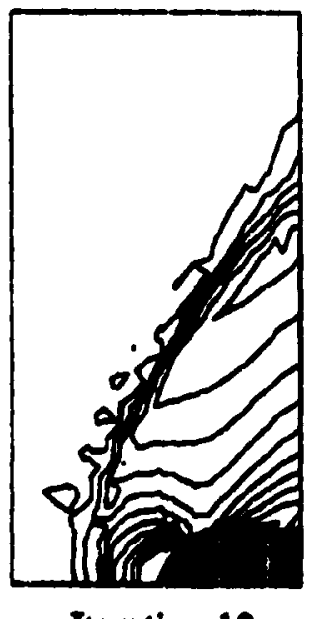

Iteration 12

Figure 7: Sencitivity Equation Molbod Itrations 


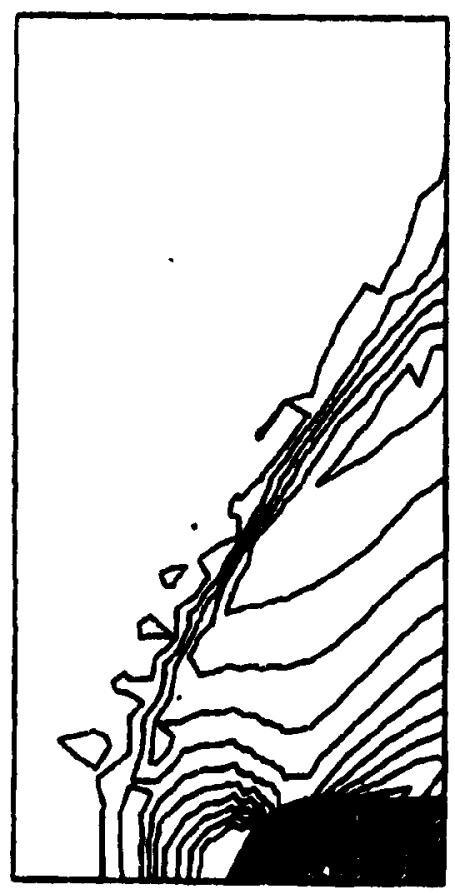

Final Converged Solution Original Long Forebody (data to be matched)

Figure 8: Comparison of Optimal Solution with Date

36 


\section{References}

[1] R. BeAM and R. F. WARMing, Journal of Computational Physics 22, 87 (1976).

[2] J. Borgganrd, J. Burns, E. Chiff, and M. Gunzburgen, Sensitivity Calculations for a 2D, Inviscid, Supereonic Forebody Problem, in Identification and Control of Systems Governed by Partial Differential Equations, edited by H. T. Banks, R. Fablano, and K. ITO, pp. 14-24, Philadelphia, PA, 1993, SIAM Publications.

[3] J. T. BorgGaARD, The Sensitivity Equation Method for Optimal Design, PhD thesis, Virginia Tech, Blackaburg, VA, 1994.

[4] J. T. BorggaARd, On the Presence of Shocks in Domain Optimization of Euler Flow;, in Flow Control, edited by M. GunzBurger, volume 68 of Proceedings of the IMA, Springer-Verlag, 1993.

[5] J. V. BuRKardT, Sersitivity Analyses and Computational Shape Optimization for Incompressible Flows, PhD thesis, Virginia Tech, Blacksburg, VA, 1995.

[6] J. A. Burns, B. B. King, and Y.-R. OH, A Computational Approach to Sensor/Actuator Location for Feedback Control of Fluid Flow Systems, in Sensing,, Actuation, and Control in Aeropropulaion, edited by J. D. PADUANo, volume 2494 of Procedings of the International Society for Optical Engineering, pp. 6069, 1995.

[7] R. G. CARten, Numerical Optimization in Hilbert Space Using Inexact Function and Gradient Evaluations, Technical Report 89-45, ICASE, 1989.

[8] R. G. CARTER, SIAM journal of Numerical Analyois 28, 251 (1991).

[9] G. K. Cooper and J. R. Sirbaugh, PARC Code: Theory and Usage, Technical Report AEDC-TR-89-15, Arnold Engineering Development Center, Arnold AFB, TN, 1989.

[10] J. E. Dennis JR., D. M. GAY, and R. E. Welsch, Trensactions on Mathematical Software 7, 348 (1981).

[11] J. E. DenNIs JR. and R. B. Scrnabel, Numerical Methods for Unconstrained Optimiration and Nonlinear Equations, Prentice Hall, Englewood Clifis, New Jersey, 1983.

[12] G. Fanun, Curves and Surfaces for Computer Aided Geometric Design: A Practical Cuide, Academic Press, San Diego, CA, 1988.

[13] P. D. Frank and G. R. Shubin, Journal of Computational Phyoice 98, 74 (1992). 
[14] E. J. Havg, K. K. Chol, and V. Komkov, Design Sensitivity Analysis of Structural Systems, volume 177 of Mathematice in Science and Engineering, Aca. demic Press, Orlando, FL, 1986.

[15] D. HuddestoN, Development of a Free-Jet Forebody Simulator Design Optimization Method, Technical Report AEDC-TR-90-22, Arnolä Engineering Development Center, Arnold AFB, TN, 1990.

[16] A. JAMEson, Journal of Scientific Computing 8, 233 (1988).

[17] A. JAMEson and J. ReUTHER, Control theory baced airfoil design using the Euler equations, in Proceedings from the 5th AIAA/USAF/NASA/ISSMO Symposium on Multidisciplinary Analysis and Optimization, pp. 206-222, 1994.

[18] C.-Y. JoH, B. Grossuan, and R. T. HaftKa, Engineering Optimization 21, 1 (1993).

[19] R. NARducci, B. Grossuan, and R. HaftKa, Inverse Problems in Engineering 2, 49 (1995).

[20] N. Pagaldipti and A. Chattopadhyay, A Discrete Semi-Analytical Procedure for Aerodynamic Sensitivity Analysis Including Grid Sensitivity, in Proceedings from the 5th AIAA/USAF/NASA/ISSMO Symposium on Multidisciplinary Analysis and Optimization, pp. 161-169, 1994.

[21] O. Pironneau, Jourmal of Fluid Mechanics 59, 117 (1973).

[22] O. Pironneav, Journal of Fluid Mechanice 60, 97 (1974).

[23] A. SHeNoY and E. M. Curf, An optimal control formulation for a flow matching problem, in Proceedings from the 5th AIAA/USAF/NASA/ISSMO Sympo. sium on Multidisciplinary Analysis and Optimization, pp. 520-528, 1994.

[24] A. C. TAYloR III, G. W. Hou, and V. M. Konvi, A Methodology for Determining Aerodynamic Sessitivity Derivatives with Respect to Variation of Geometric Shape, in Proceedings of the AIAA/ASME/ASCE/AHS/ASC s2nd Structures, Structural Dyramics, and Materials Conference, Baltimore, MD, 1991, AIAA paper 91-1101.

[25] A. C. Taylor III, G. W. Hov, and V. M. KorIVI, Seositivity Analysie, Approximate Analyais and Design Optimization for Internal and External Viscous Flows, in Praceedings of AIAA Aircraft Design Systems and Operations Meeting, 1991, AIA A paper 91-3083.

[26] J. Thompson, Z. Warsi, and C. Mastin, Numerical Grid Generation: foundations and applicatione, North-Holland Publishing Company, New York, 1985. 


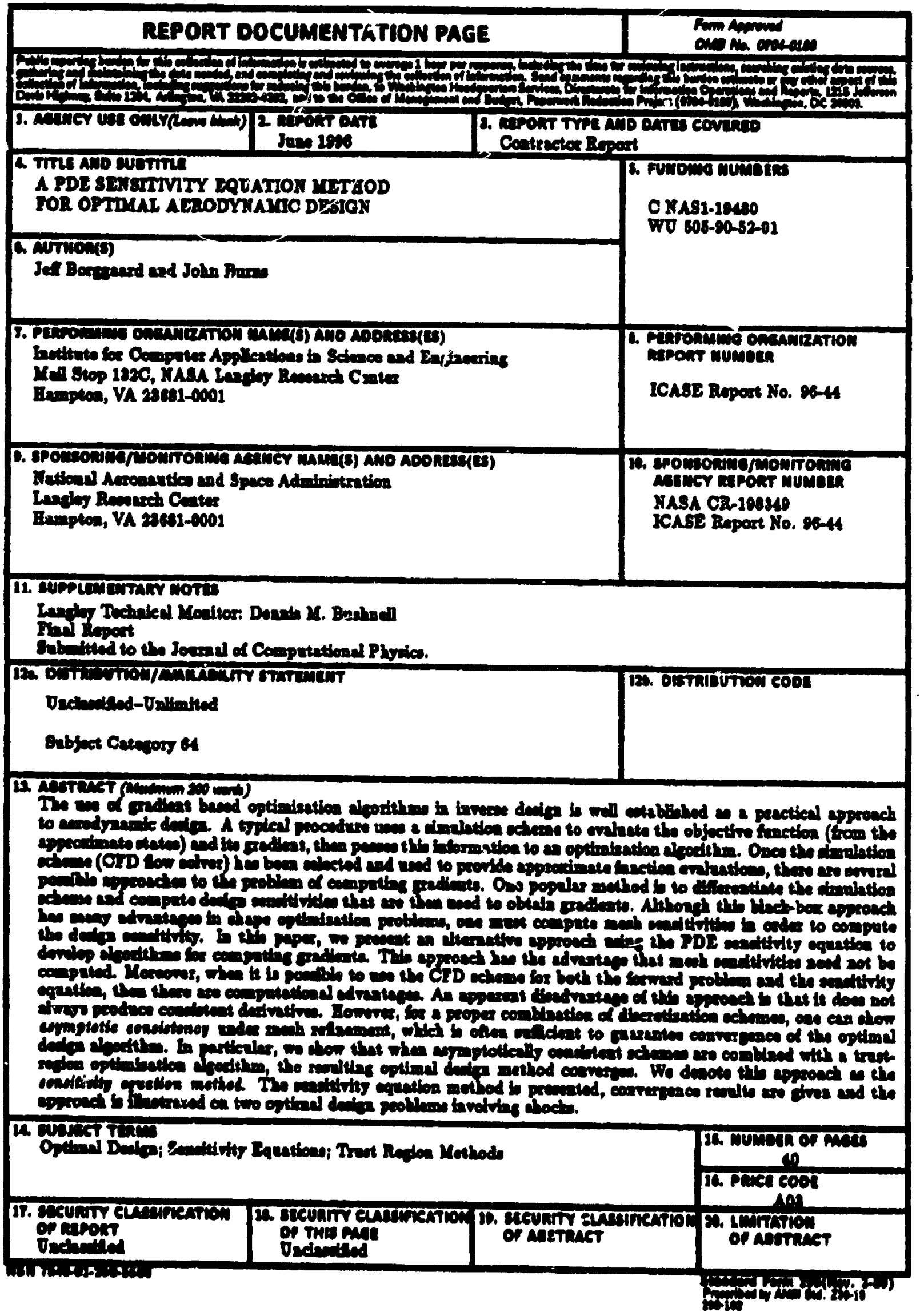

